\title{
Dual Taylor Series, Spline Based Function and Integral Approximation and Applications
}

\author{
Roy M. Howard $1 D$
}

School of Electrical Engineering, Computing and Mathematical Sciences, Curtin University, GPO Box U1987, Perth 6845, Australia; r.howard@curtin.edu.au

Received: 2 March 2019; Accepted: 25 March 2019; Published: 1 April 2019

\begin{abstract}
In this paper, function approximation is utilized to establish functional series approximations to integrals. The starting point is the definition of a dual Taylor series, which is a natural extension of a Taylor series, and spline based series approximation. It is shown that a spline based series approximation to an integral yields, in general, a higher accuracy for a set order of approximation than a dual Taylor series, a Taylor series and an antiderivative series. A spline based series for an integral has many applications and indicative examples are detailed. These include a series for the exponential function, which coincides with a Padé series, new series for the logarithm function as well as new series for integral defined functions such as the Fresnel Sine integral function. It is shown that these series are more accurate and have larger regions of convergence than corresponding Taylor series. The spline based series for an integral can be used to define algorithms for highly accurate approximations for the logarithm function, the exponential function, rational numbers to a fractional power and the inverse sine, inverse cosine and inverse tangent functions. These algorithms are used to establish highly accurate approximations for $\pi$ and Catalan's constant. The use of sub-intervals allows the region of convergence for an integral approximation to be extended.
\end{abstract}

Keywords: integral approximation; function approximation; Taylor series; dual Taylor series; spline approximation; antiderivative series; exponential function; logarithm function; Fresnel sine integral; Padé series; Catalan's constant

MSC: 26A06; 26A36; 33B10; 41A15; 41A58

\section{Introduction}

The mathematics underpinning dynamic behaviour is of fundamental importance to modern science and technology and integration theory is foundational. The history of integration dates from early recorded history, with area calculations being prominent, e.g., [1,2]. The modern approach to integration commences with Newton and Leibniz and Thomson [3,4] provides a lucid, and up to date, perspective on the approaches of Newton, Riemann and Lebesgue and the more recent work of Henstock and Kurzweil.

A useful starting point for integration theory is the second part of the Fundamental Theorem of Calculus which states

$$
\int_{\alpha}^{\beta} f(t) d t=F(\beta)-F(\alpha),
$$

where $f=F^{(1)}$ on $[\alpha, \beta]$ for some antiderivative function $F$, assuming $f$ is integrable, e.g., [4-6]. However, within all frameworks of integration, a practical problem is to determine antiderivative functions for, or suitable analytical approximations to, specified integrals. Despite the impressive collection of results that can be found in tables books such as Gradsteyn and Ryzhik [7], the problem of 
determining an antiderivative function, or an approximation to the integral of a specified function, in general, is problematic. As a consequence, numerical evaluation of integrals is widely used. The problem is: For an arbitrary class of functions $\mathfrak{J}$, and a specified interval $[\alpha, \beta]$, how to determine an analytical expression, or analytical approximation, to the integral

$$
I(t)=\int_{\alpha}^{t} f(\lambda) d \lambda, f \in \mathfrak{J}, t \in[\alpha, \beta] .
$$

Approaches include use of integration by parts, e.g., [8], use of Taylor series, asymptotic expansions, e.g., $[9,10]$, etc.

A useful approximation approach is to use uniform convergence, bounded convergence, monotone convergence or dominated convergence of a sequence of functions and a representative statement arising from dominated convergence, e.g., [11], is: If $\left\{f_{i}\right\}$ is a sequence of Lebesgue integrable functions on $[\alpha, \beta], \lim _{i \rightarrow \infty} f_{i}(t)=f(t)$ pointwise almost everywhere on $[\alpha, \beta]$, and there exists a Lebesgue integrable function $g$ on $[\alpha, \beta]$ with the property $\left|f_{i}(t)\right|<|g(t)|, t \in[\alpha, \beta]$ almost everywhere and for all $i$, then

$$
\lim _{i \rightarrow \infty} \int_{\alpha}^{t} f_{i}(\lambda) d \lambda=\int_{\alpha}^{t} f(\lambda) d \lambda=\lim _{i \rightarrow \infty} F_{i}(t), t \in[\alpha, \beta],
$$

where

$$
F_{i}(t)=\int_{\alpha}^{t} f_{i}(\lambda) d \lambda
$$

For the case where $\left\{f_{i}\right\}_{i=1}^{\infty}$ is such that the corresponding sequence of antiderivative functions $\left\{F_{i}\right\}_{i=1}^{\infty}$ is known, then an analytical approximation to the integral of $f$ is defined by $F_{n}(t)$. It is well known that polynomial, trigonometric and orthogonal functions can be defined to approximate a specified function, e.g., [12]. In general, such approximations require knowledge of the function at a specified number of points within the approximating interval and the use of approximating functions based on points within the region of integration underpins numerical evaluation of integrals, e.g., [13]. Of interest is if function values at the end point of an interval, alone, can suffice to provide a suitable analytic approximation to a function and its integral. In this context, the use of a Taylor series approximation is one possible approach but, in general, the approximation has a limited region of convergence. The region of convergence can be extended through use of a dual Taylor series which is introduced in this paper and is based on utilizing two demarcation functions. An alternative approach consider in this paper is to use spline based approximations.

It is shown that a spline based integral approximation, based solely on function values at the interval endpoints, has a simple analytic form and, in general, better convergence that an integral approximation based on a Taylor or a dual Taylor series. Further, a spline based integral approximation leads to new series for many defined integral functions, as well as many standard functions, with, in general, better convergence than a Taylor series. The spline based series for an integral can be used to define algorithms for highly accurate approximations for specific functions and new results for definite integrals are shown. As is usual, interval sub-division leads to improved integral accuracy and high levels of precision in results can readily be obtained.

In Section 2, a brief introduction is provided for integral approximation based on an antiderivative series and a Taylor series. A natural generalization of a Taylor series is a dual Taylor series and this is defined in Section 3 along with its application to integral approximation. An alternative to a dual Taylor series approximation to a function is a spline based approximation and this is detailed in Section 4 along with its application to integral approximation. A comparison of the antiderivative, Taylor series, dual Taylor series and spline approaches for integral approximation is detailed in Section 5. It is 
shown that a spline based approach, in general, is superior. Applications of a spline based integral approximation are detailed in Section 6 and concluding comments are detailed in Section 7.

In terms of notation $f^{(k)}(t)=\frac{d^{k}}{d t^{k}} f(t)$ is used and all derivatives in the paper are with respect to the variable $t$.

Mathematica has been used to generate all numerical results, graphic display of results and, where appropriate, analytical results.

\section{Integral Approximation: Antiderivative Series, Taylor Series}

For integral approximation over a specified interval, and based on function values at the interval endpoints, two standard results can be considered: First, an antiderivative series based on integration by parts. Second, a Taylor series expansion of an integral.

\subsection{Antiderivative Series}

An antiderivative series for an integral can be established by application of integration by parts, e.g., [14]: If $f: R \rightarrow R$ is $n$th order differentiable on a closed interval [ $\alpha, t]$ (left and right hand limits, as appropriate, at $\alpha, t)$ then

$$
I(t)=\int_{\alpha}^{t} f(\lambda) d \lambda=\sum_{k=0}^{n-1} c_{k}\left[\alpha^{k+1} f^{(k)}(\alpha)-t^{k+1} f^{(k)}(t)\right]+R_{n}(\alpha, t),
$$

where

$$
c_{k}=\frac{(-1)^{k+1}}{(k+1) !}, \quad R_{n}(\alpha, t)=\frac{(-1)^{n}}{n !} \cdot \int_{\alpha}^{t} \lambda^{n} f^{(n)}(\lambda) d \lambda .
$$

\subsection{Taylor Series Integral Approximation}

A Taylor series based approximation to an integral is based on a Taylor series function approximation which dates from 1715 [15]. Consider an interval $[\alpha, \beta]$ and a function $f: \boldsymbol{R} \rightarrow \boldsymbol{R}$ whose derivatives of all orders up to, and including, $n+1$ exist at all points in the interval $[\alpha, \beta]$. A $n$th order Taylor series of a function $f$, and based on the point $\alpha$, is defined according to

$$
\begin{aligned}
f_{n}(\alpha, t) & =f(\alpha)+\sum_{k=1}^{n} \frac{(t-\alpha)^{k} f^{(k)}(\alpha)}{k !} \\
& =\sum_{k=0}^{n} \frac{(t-\alpha)^{k} f^{(k)}(\alpha)}{k !}, \quad t \neq \alpha .
\end{aligned}
$$

For notational simplicity, it is useful to use the latter form of the definition with the former form being implicit for the case of $t=\alpha$. A Taylor series enables a function $f: \boldsymbol{R} \rightarrow \boldsymbol{R}$, assumed to be $(n+1)$ th order differentiable, to be written as

$$
f(t)=f_{n}(\alpha, t)+R_{n}(\alpha, t), \quad t \in(\alpha, \beta),
$$

where an explicit expression for the remainder function $R_{n}(\alpha, t)$ is

$$
R_{n}(\alpha, t)=\int_{\alpha}^{t} \frac{(t-\lambda)^{n} f^{(n+1)}(\lambda)}{n !} d \lambda
$$

See, for example, [8] or [16] for a proof. A sufficient condition for convergence of a Taylor series is for

$$
\lim _{n \rightarrow \infty} \frac{f_{\max }^{(n)}(t-\alpha)^{n}}{n !}=0,
$$


where $f_{\max }^{(n)}=\sup \left\{f^{(n)}(t): t \in[\alpha, \beta]\right\}$.

It then follows that a $n$th order Taylor series for the integral

$$
I(t)=\int_{\alpha}^{t} f(\lambda) d \lambda
$$

based on the point $\alpha$, is

$$
I(t)=\sum_{k=0}^{n-1} c_{k}(t-\alpha)^{k+1} f^{(k)}(\alpha)+R_{n}(\alpha, t)
$$

where

$$
c_{k}=\frac{1}{(k+1) !}, \quad R_{n}(\alpha, t)=\int_{\alpha}^{t} \frac{(t-\lambda)^{n} f^{(n)}(\lambda)}{n !} d \lambda .
$$

\section{Dual Taylor Series}

A natural generalization of a Taylor series is to use two Taylor series, based at different points, and to combine them by using appropriate weighting, or demarcation, functions. The result is a dual Taylor series.

\subsection{Demarcation Functions}

For the normalized case of an interval [0, 1], a dual Taylor series requires two demarcations functions, denoted $m_{N}$ and $q_{N}$, which have the monotonic decreasing/increasing form illustrated in Figure 1. The ideal, and normalized, demarcation function are defined according to

$$
m_{N}(t)=\left\{\begin{array}{cc}
1 & 0<t<\frac{1}{2} \\
0.5 & t=0.5 \\
0 & \frac{1}{2}<t<1
\end{array} \quad q_{N}(t)=m_{N}(1-t)=\left\{\begin{array}{lr}
0 & 0<t<\frac{1}{2} \\
0.5 & t=0.5 \\
1 & \frac{1}{2}<t<1
\end{array}\right.\right.
$$

and are such that

$$
m_{N}(t)+q_{N}(t)=1 .
$$

Whether idealized or not, the assumption is made that $m_{N}$ and $q_{N}$ are such that (15) is satisfied. Further, for the case where $m_{N}$ is antisymmetrical around the point $(1 / 2,1 / 2)$, it follows that $m_{N}(t)=$ $1-m_{N}(1-t)$ and $q_{N}(t)=m_{N}(1-t)$. This is assumed.

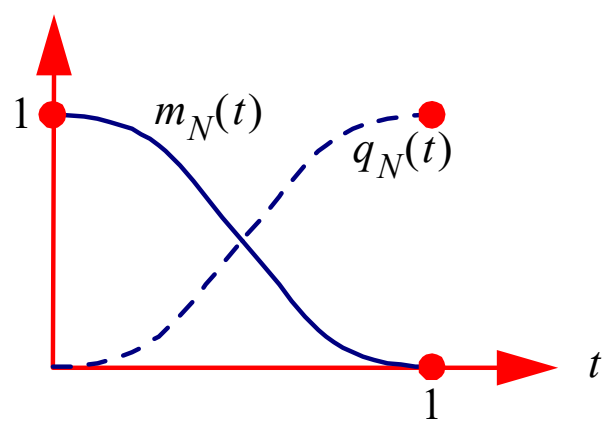

Figure 1. Illustration of the normalized demarcation functions $m_{N}(t)$ and $q_{N}(t)$.

For a dual Taylor series, the further requirement is that the demarcation functions do not affect the value of the derivatives of the series at the end points of the interval being considered. This can be achieved by the further constraints of the right and left hand derivatives, of all orders, being zero, 
respectively, at the points zero and one, i.e., $m_{N}^{(k)}\left(0^{+}\right)=q_{N}^{(k)}\left(0^{+}\right)=0$ and $m_{N}^{(k)}\left(1^{-}\right)=q_{N}^{(k)}\left(1^{-}\right)=0$ for $k \in\{1,2, \ldots\}$.

An example of a suitable demarcation function is

$$
m_{N}(t)=\left\{\begin{array}{cl}
1 & t=0 \\
\frac{1}{2}\left[1-\tanh \left[\frac{k_{D}(2 t-1)}{1-(2 t-1)^{2}}\right]\right] & 0<t<1 \\
0 & t=1
\end{array}\right.
$$

and the graph of this function is shown in Figure 2 for the case of $k_{D} \in\{1,2,5,10\}$.

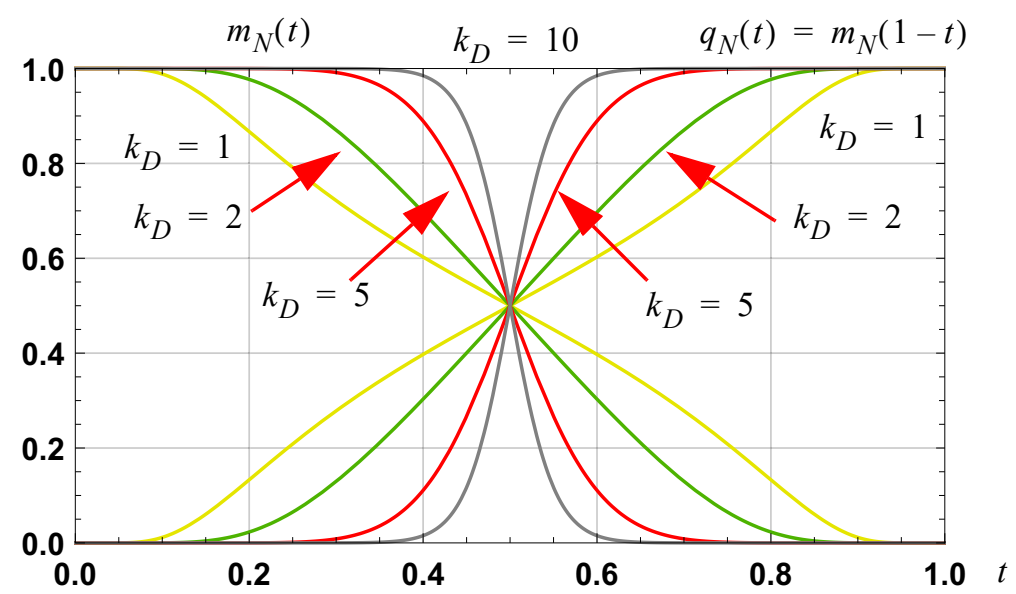

Figure 2. Graph of the normalized demarcation functions $m_{N}(t)$ and $q_{N}(t)$ as defined by (16). Such functions are infinitely differentiable on $(0,1)$, and have right and left hand derivatives, of all orders, that are zero, respectively, at the points of zero and one.

For the denormalized case, and for the interval $[\alpha, \beta]$, the demarcation functions are defined according to

$$
m(t)=m_{N}\left(\frac{t-\alpha}{\beta-\alpha}\right), \quad q(t)=q_{N}\left(\frac{t-\alpha}{\beta-\alpha}\right)=m_{N}\left(\frac{\beta-t}{\beta-\alpha}\right) .
$$

\section{Polynomial Based Demarcation Function}

Polynomial demarcation functions are of interest because, if they are associated with a Taylor series, the resulting composite function has a known antiderivative form.

A normalized polynomial based demarcation function, of order $n$, and for the interval $[0,1]$, is the $(2 n+1)$ th order polynomial

$$
\begin{aligned}
m_{N, n}(t) & =(1-t)^{n+1} \cdot\left[1+\sum_{k=1}^{n} \frac{(n+k) !}{n ! \cdot k !} \cdot t^{k}\right] \\
& =(1-t)^{n+1} \cdot \sum_{k=0}^{n} \frac{(n+k) !}{n ! \cdot k !} \cdot t^{k}, \quad t \neq 0 .
\end{aligned}
$$

For notational simplicity, the latter form is used with the former form being implicit for the case of $t=0$. This function satisfies the constraints

$$
m_{N, n}(0)=1, \quad m_{N, n}(1)=0,\left.\quad m_{N, n}^{(k)}(t)\right|_{t \in\{0,1\}}=0, \quad k \in\{1, \ldots, n\},
$$


and is antisymmetric around the point $(1 / 2,1 / 2)$. The associated quadrature polynomial demarcation function is

$$
q_{N, n}(t)=1-m_{N, n}(t)=m_{N, n}(1-t)=t^{n+1} \cdot \sum_{k=0}^{n} \frac{(n+k) !}{n ! \cdot k !} \cdot(1-t)^{k}
$$

To derive (18), a useful approach is to solve for the coefficients of a $(2 n+1)$ th order polynomial, subject to the constraints specified by (19), and starting with the case of $n=1,2, \ldots$. The coefficient form of $\frac{(n+k) !}{n ! \cdot k !}$ in (18) can be inferred from the results specified in Pascal's triangle.

An alternative form for $m_{N, n}(t)$ is

$$
\begin{aligned}
m_{N, n}(t) & =1+(n+1) \sum_{u=0}^{n+1} \sum_{v=0, u+v \neq 0}^{n} \frac{(-1)^{u} \cdot(n+v) !}{(n+1-u) ! \cdot u ! \cdot v !} \cdot t^{u+v} \\
& =(\mathrm{n}+1) \sum_{u=0}^{n+1} \sum_{v=0}^{n} \frac{(-1)^{u} \cdot(n+v) !}{(n+1-u) ! \cdot u ! \cdot v !} \cdot t^{u+v}, \quad t \neq 0,
\end{aligned}
$$

which arises from using the binomial formula on $(1-t)^{n+1}$. For notational simplicity, the latter form is used with the former form being implicit for the case of $t=0$.

The graphs of the polynomial based demarcation functions are shown in Figure 3. For the denormalized case, and for the interval $[\alpha, \beta]$, the demarcation functions are defined according to

$$
m_{n}(t)=m_{N, n}\left(\frac{t-\alpha}{\beta-\alpha}\right), \quad q_{n}(t)=q_{N, n}\left(\frac{t-\alpha}{\beta-\alpha}\right)=m_{N, n}\left(\frac{\beta-t}{\beta-\alpha}\right) .
$$

Explicit forms for the demarcation functions, based on the form specified in (18), are:

$$
\begin{aligned}
& m_{n}(t)=\left[\frac{\beta-t}{\beta-\alpha}\right]^{n+1}\left[1+\sum_{k=1}^{n} \frac{(n+k) !}{n ! \cdot k !} \cdot\left[\frac{t-\alpha}{\beta-\alpha}\right]^{k}\right]=\left[\frac{\beta-t}{\beta-\alpha}\right]^{n+1} \sum_{k=0}^{n} \frac{(n+k) !}{n ! \cdot k !} \cdot\left[\frac{t-\alpha}{\beta-\alpha}\right]^{k} \\
& q(t)=\left[\frac{t-\alpha}{\beta-\alpha}\right]^{n+1}\left[1+\sum_{k=1}^{n} \frac{(n+k) !}{n ! \cdot k !} \cdot\left[\frac{\beta-t}{\beta-\alpha}\right]^{k}\right]=\left[\frac{t-\alpha}{\beta-\alpha}\right]^{n+1} \sum_{k=0}^{n} \frac{(n+k) !}{n ! \cdot k !} \cdot\left[\frac{\beta-t}{\beta-\alpha}\right]^{k} .
\end{aligned}
$$

The second form in these equations are valid, respectively, for $t \neq \alpha$ and $t \neq \beta$, and for notational simplicity, are used.

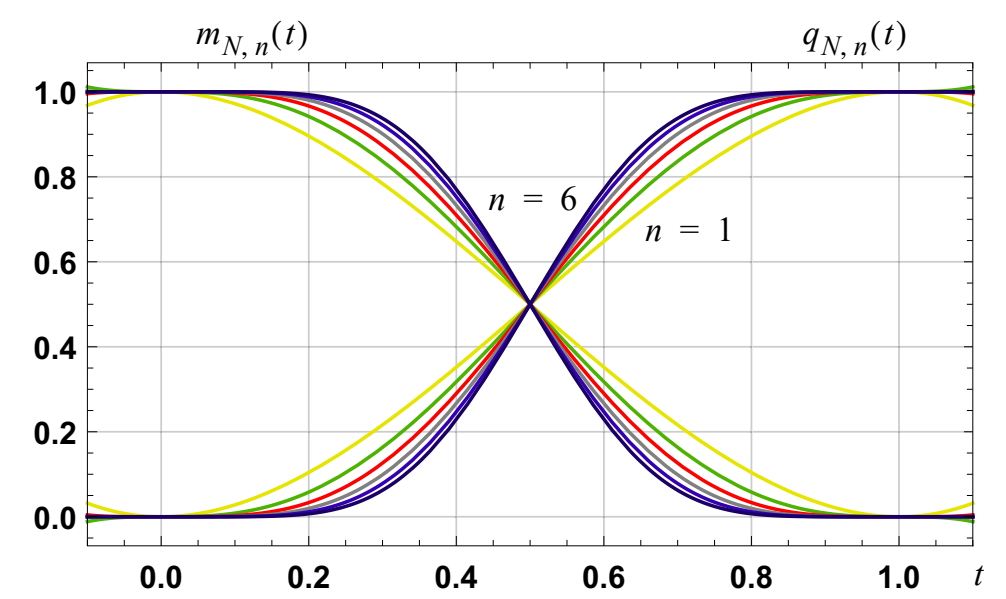

Figure 3. Graph of the normalized polynomial demarcation functions, of orders one to six, for the interval $[0,1]$. 


\subsection{Dual Taylor Series}

For the interval $[\alpha, \beta]$, a dual Taylor series, of order $n$, is the weighted summation of two $n$th order Taylor series, one based at $\alpha$ and one at $\beta$, and is defined according to

$$
\begin{aligned}
f_{n}(\alpha, \beta, t)= & m(t)\left[f(\alpha)+(t-\alpha) f^{(1)}(\alpha)+\frac{(t-\alpha)^{2} f^{(2)}(\alpha)}{2}+\cdots+\frac{(t-\alpha)^{n} f^{(n)}(\alpha)}{n !}\right]+ \\
& q(t)\left[f(\beta)+(t-\beta) f^{(1)}(\beta)+\frac{(t-\beta)^{2} f^{(2)}(\beta)}{2}+\cdots+\frac{(t-\beta)^{n} f^{(n)}(\beta)}{n !}\right], t \in[\alpha, \beta]
\end{aligned}
$$

where $m$ and $q$ are the demarcation functions defined by (17). Using the Taylor series notation as specified in (7):

$$
f_{n}(\alpha, \beta, t)=m(t) f_{n}(\alpha, t)+q(t) f_{n}(\beta, t) .
$$

By construction:

$$
\begin{gathered}
f_{n}(\alpha, \beta, \alpha)=f(\alpha), \quad f_{n}(\alpha, \beta, \beta)=f(\beta), \\
f_{n}^{(k)}(\alpha, \beta, \alpha)=f^{(k)}(\alpha), \quad f_{n}^{(k)}(\alpha, \beta, \beta)=f^{(k)}(\beta), 1 \leq k \leq n .
\end{gathered}
$$

For the case of polynomial demarcation functions, $m(t)=m_{n}(t)$ and $q(t)=q_{n}(t)$, as specified by (23), and an explicit expression for $f_{n}(\alpha, \beta, t)$ is

$$
\begin{aligned}
f_{n}(\alpha, \beta, t)= & m_{n}(t) f_{n}(\alpha, t)+q_{n}(t) f_{n}(\beta, t) \\
=\quad & {\left[\frac{\beta-t}{\beta-\alpha}\right]^{n+1} \sum_{k=0}^{n} \frac{(n+k) !}{n ! \cdot k !} \cdot\left[\frac{t-\alpha}{\beta-\alpha}\right]^{k} \cdot \sum_{k=0}^{n} \frac{(t-\alpha)^{k} f^{(k)}(\alpha)}{k !}+} \\
& {\left[\frac{t-\alpha}{\beta-\alpha}\right]^{n+1} \sum_{k=0}^{n} \frac{(n+k) !}{n ! \cdot k !} \cdot\left[\frac{\beta-t}{\beta-\alpha}\right]^{k} \cdot \sum_{k=0}^{n} \frac{(t-\beta)^{k} f^{(k)}(\beta)}{k !} . }
\end{aligned}
$$

A dual Taylor series allows a function to be written as

$$
f(t)=f_{n}(\alpha, \beta, t)+R_{n}(\alpha, \beta, t), \quad t \in(\alpha, \beta),
$$

where the remainder function $R_{n}(\alpha, \beta, t)$ is defined according to

$$
R_{n}(\alpha, \beta, t)=m(t) \int_{\alpha}^{t} \frac{(t-\lambda)^{n} f^{(n+1)}(\lambda)}{n !} d \lambda-q(t) \int_{t}^{\beta} \frac{(t-\lambda)^{n} f^{(n+1)}(\lambda)}{n !} d \lambda, t \in[\alpha, \beta] .
$$

The proof of this result is detailed in Appendix A.

\subsubsection{Convergence}

With the definition of $f_{\max }^{(n)}=\sup \left\{f^{(n)}(t): t \in[\alpha, \beta]\right\}$, it follows that a bound on the remainder function is

$$
\left|R_{n}(\alpha, \beta, t)\right| \leq \frac{f_{\max }^{(n+1)}}{(n+1) !} \cdot\left[m(t) \cdot(t-\alpha)^{n+1}+q(t) \cdot(\beta-t)^{n+1}\right] .
$$

It then follows, from the nature of the demarcation functions, that a sufficient condition for the convergence of a dual Taylor series for the interval $[\alpha, \beta]$ is for

$$
\lim _{n \rightarrow \infty} \frac{f_{\max }^{(n)}(\beta-\alpha)^{n}}{n !}=0, f_{\max }^{(n)}=\sup \left\{f^{(n)}(t): t \in[\alpha, \beta]\right\} .
$$


For ideal demarcation functions, where the Taylor series based at $\alpha$ only has influence on the interval $\left[\alpha, \frac{\alpha+\beta}{2}\right]$, a sufficient condition for convergence is for

$$
\lim _{n \rightarrow \infty} \frac{f_{\max }^{(n)}(\beta-\alpha)^{n}}{2^{n} \cdot n !}=0 .
$$

\subsubsection{Example}

Consider the function

$$
f(t)=\tanh (t)+\exp (-t) \sin \left(\pi t^{2}\right) .
$$

Dual Taylor series approximations to this function, of orders 4, 6, 8, 10, for the interval $[0,1]$ and defined by (27), are shown in Figure 4 using the polynomial demarcation functions specified by (23).

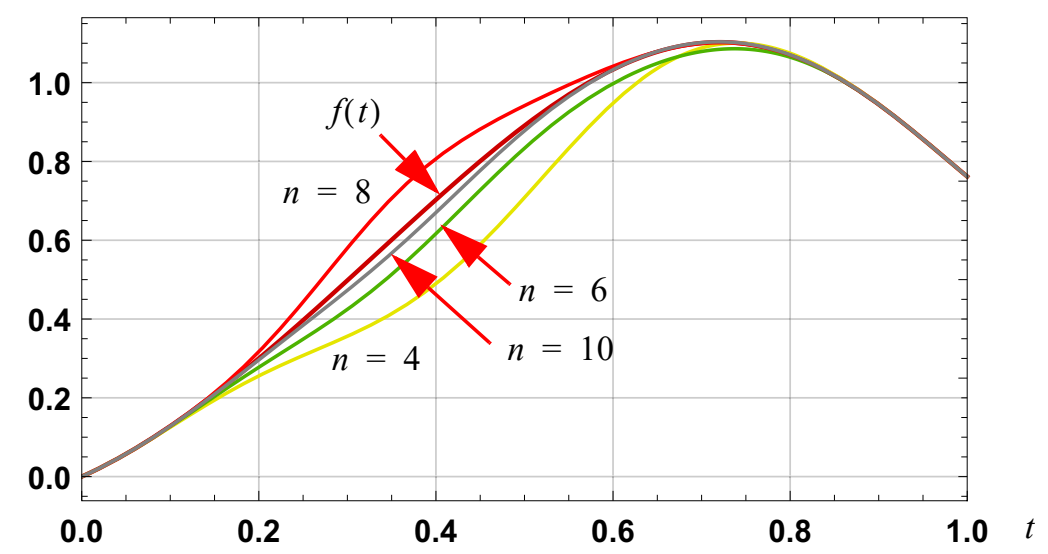

Figure 4. Graph of the 4th, 6th, 8th and 10th order dual Taylor series approximations, based on the interval $[0,1]$, for the function defined by (33).

\subsection{Integral Approximation}

With a polynomial demarcation function, the integral of a dual Taylor series is well defined and leads to the following equality

$$
I(t)=\int_{\alpha}^{t} f(\lambda) d \lambda=\sum_{k=0}^{n} c_{n, k}(t-\alpha)^{k+1}\left[f^{(k)}(\alpha)+(-1)^{k} f^{(k)}(t)\right]+R_{n}(\alpha, t),
$$

where

$$
c_{n, k}=\frac{n+1}{k !} \cdot \sum_{u=0}^{n+1} \frac{(-1)^{u}}{u ! \cdot(n+1-u) !} \cdot\left[\sum_{v=0}^{n} \frac{(n+v) !}{v !} \cdot \frac{1}{u+v+k+1}\right]
$$

and

$$
\begin{aligned}
R_{n}^{(1)}(\alpha, t)= & c_{n, 0}[f(t)-f(\alpha)]-\sum_{k=1}^{n} c_{n, k}(k+1)(t-\alpha)^{k} f^{(k)}(\alpha)+ \\
& \sum_{k=1}^{n}(-1)^{k+1}(t-\alpha)^{k} f^{(k)}(t)\left[(k+1) c_{n, k}-c_{n, k-1}\right]+c_{n, n}(-1)^{n+1}(t-\alpha)^{n+1} f^{(n+1)}(t)
\end{aligned}
$$

The proof is detailed in Appendix B.

\section{Spline Approximation}

A $n$th order spline approximation, $f_{n}$, on the interval $[\alpha, \beta]$, to a function $f$, which is differentiable up to order $n$, is a $(2 n+1)$ th order polynomial that equals the function, in terms of value and derivatives 
up to order $n$, at the end points of the interval. A $n$th order spline function for the interval $[\alpha, \beta]$, thus, has the form

$$
f_{n}(\alpha, \beta, t)=c_{0}+c_{1} t+c_{2} t^{2}+\cdots+c_{2 n+1} t^{2 n+1}, t \in[\alpha, \beta],
$$

where the coefficients $c_{0}, \ldots, c_{2 n+1}$ are such that the function and the spline approximation, as well as their derivatives of order $1, \ldots, n$, take on the same values at the end points of the interval, i.e.,

$$
f^{(k)}(t)=\left.f_{n}^{(k)}(\alpha, \beta, t)\right|_{t \in\{\alpha, \beta\}}, k \in\{0,1, \cdots, n\} .
$$

The case of $n=1$ corresponds to a cubic spline.

The use of the following symmetrical form

$$
\begin{aligned}
f_{n}(\alpha, \beta, t)= & h_{n}(t)\left[a_{00}+a_{01}(t-\alpha)+a_{02}(t-\alpha)^{2}+\cdots+a_{0 n}(t-\alpha)^{n}\right] f(\alpha)+ \\
& h_{n}(t)(t-\alpha)\left[a_{10}+a_{11}(t-\alpha)+a_{12}(t-\alpha)^{2}+\cdots+a_{1, n-1}(t-\alpha)^{n-1}\right] f^{(1)}(\alpha)+ \\
& \cdots \\
& h_{n}(t)(t-\alpha)^{n-1}\left[a_{n-1,0}+a_{n-1,1}(t-\alpha)\right] f^{(n-1)}(\alpha)+ \\
& h_{n}(t)(t-\alpha)^{n}\left[a_{n 0}\right] f^{(n)}(\alpha)+ \\
& g_{n}(t)\left[b_{00}+b_{01}(t-\beta)+b_{02}(t-\beta)^{2}+\cdots+b_{0 n}(t-\beta)^{n}\right] f(\beta)+ \\
& g_{n}(t)(t-\beta)\left[b_{10}+b_{11}(t-\beta)+b_{12}(t-\beta)^{2}+\cdots+b_{1, n-1}(t-\beta)^{n-1}\right] f^{(1)}(\beta)+ \\
& \cdots \\
& g_{n}(t)(t-\beta)^{n-1}\left[b_{n-1,0}+b_{n-1,1}(t-\beta)\right] f^{(n-1)}(\beta)+ \\
& g_{n}(t)(t-\beta)^{n}\left[b_{n 0}\right] f^{(n)}(\beta)
\end{aligned}
$$

where $h_{n}(t)=\frac{(t-\beta)^{n+1}}{(\beta-\alpha)^{n+1}}$ and $\mathrm{g}_{n}(t)=\frac{(t-\alpha)^{n+1}}{(\beta-\alpha)^{n+1}}$, allows the sequential solving of the unknown coefficients and leads to the result (see Appendix $C$ ):

$$
\begin{aligned}
f_{n}(\alpha, \beta, t)= & \frac{(\beta-t)^{n+1}}{(\beta-\alpha)^{n+1}} \cdot \sum_{k=0}^{n} \frac{(t-\alpha)^{k}}{k !} \cdot f^{(k)}(\alpha) \cdot \sum_{i=0}^{n-k} \frac{(n+i) !}{i ! n !} \cdot \frac{(t-\alpha)^{i}}{(\beta-\alpha)^{i}}+ \\
& \frac{(t-\alpha)^{n+1}}{(\beta-\alpha)^{n+1}} \cdot \sum_{k=0}^{n} \frac{(-1)^{k}(\beta-t)^{k}}{k !} \cdot f^{(k)}(\beta) \cdot \sum_{i=0}^{n-k} \frac{(n+i) !}{i ! \cdot n !} \cdot \frac{(\beta-t)^{i}}{(\beta-\alpha)^{i}}
\end{aligned}
$$

This expression can be written in the following manner which is similar in form to that of a dual Taylor series:

$$
\begin{aligned}
f_{n}(\alpha, \beta, t)= & m_{n}(t) \cdot \sum_{k=0}^{n} \frac{(t-\alpha)^{k}}{k !} \cdot f^{(k)}(\alpha) \cdot\left[1+c_{n, k}(t)\right]+ \\
& q_{n}(t) \cdot \sum_{k=0}^{n} \frac{(t-\beta)^{k}}{k !} \cdot f^{(k)}(\beta) \cdot\left[1+d_{n, k}(t)\right]
\end{aligned}
$$

where $m_{n}(t)$ and $q_{n}(t)$ are the denormalized polynomial demarcation functions defined by (23) and the coefficient functions are defined according to $c_{n, 0}(t)=d_{n, 0}(t)=0$ and

$$
c_{n, k}(t)=\frac{-\left[\sum_{i=n-k+1}^{n} \frac{(n+i) !}{i ! \cdot n !} \cdot \frac{(t-\alpha)^{i}}{(\beta-\alpha)^{i}}\right]}{\sum_{i=0}^{n} \frac{(n+i) !}{i ! \cdot n !} \cdot \frac{(t-\alpha)^{i}}{(\beta-\alpha)^{i}}}, \quad d_{n, k}(t)=\frac{-\left[\sum_{i=n-k+1}^{n} \frac{(n+i) !}{i ! \cdot n !} \cdot \frac{(\beta-t)^{i}}{(\beta-\alpha)^{i}}\right]}{\sum_{i=0}^{n} \frac{(n+i) !}{i ! \cdot n !} \cdot \frac{(\beta-t)^{i}}{(\beta-\alpha)^{i}}}, k \in\{1, \cdots, n\}
$$

The proof of these results is detailed in Appendix $C$.

The coefficient functions are such that $-1<c_{n, k}(t), d_{n, k}(t)<0$ for $k \in\{1, \ldots, n\}$ and a comparison of (41) with (27) shows that a spline approximation converges to a dual Taylor approximation when $c_{n, k}(t)$ and $d_{n, k}(t)$ converge to zero. The variation in $1+c_{n, k}(t)$ is illustrated in Figure 5. 


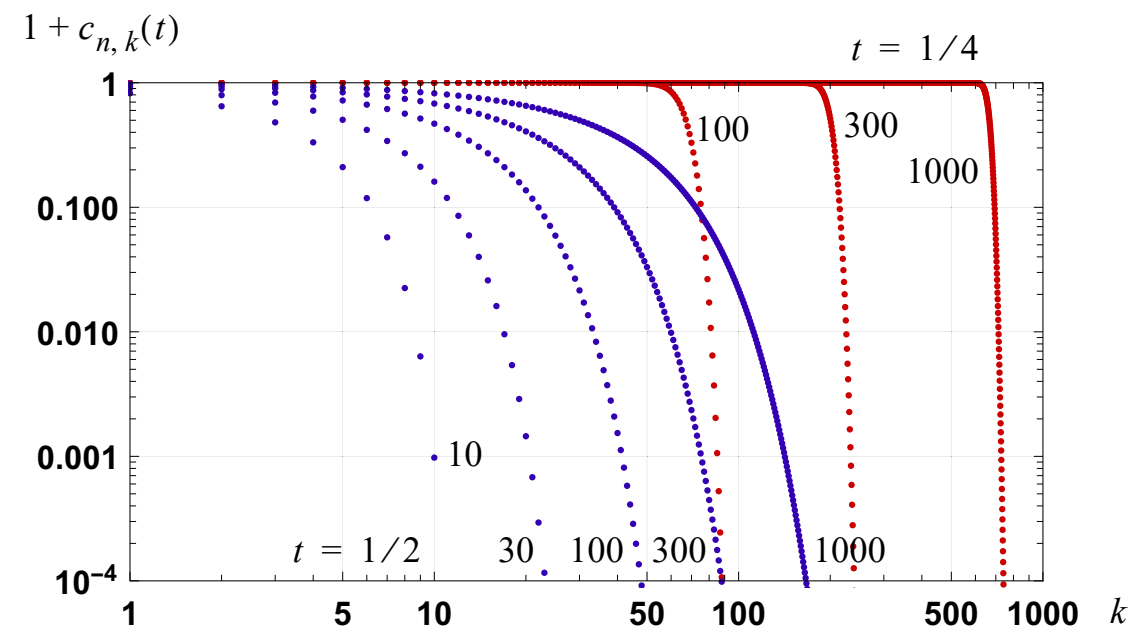

Figure 5. Graph of $1+c_{n, k}(t)$, for the case of $\alpha=0$ and $\beta=1$ and for $t=1 / 4$ and $t=1 / 2$. Results are shown for $n \in\{10,30,100,300,1000\}$ when $t=1 / 2$ and $n \in\{100,300,1000\}$ when $t=1 / 4$.

\subsection{Examples}

The zeroth to third order spline approximations to a function $f$ for the interval $[\alpha, \beta]$ are:

$$
\begin{gathered}
f_{0}(\alpha, \beta, t)=\frac{\beta-t}{\beta-\alpha} \cdot f(\alpha)+\frac{t-\alpha}{\beta-\alpha} \cdot f(\beta) \\
f_{1}(\alpha, \beta, t)=\frac{(\beta-t)^{2}}{(\beta-\alpha)^{2}} \cdot\left[f(\alpha)\left[1+\frac{2(t-\alpha)}{\beta-\alpha}\right]+(t-\alpha) \cdot f^{(1)}(\alpha)\right]+\frac{(t-\alpha)^{2}}{(\beta-\alpha)^{2}} \cdot\left[f(\beta)\left[1+\frac{2(\beta-t)}{\beta-\alpha}\right]+(t-\beta) \cdot f^{(1)}(\beta)\right] \\
f_{2}(\alpha, \beta, t)=\frac{(\beta-t)^{3}}{(\beta-\alpha)^{3}} \cdot\left[f(\alpha)\left[1+\frac{3(t-\alpha)}{\beta-\alpha}+\frac{6(t-\alpha)^{2}}{(\beta-\alpha)^{2}}\right]+(t-\alpha) \cdot f^{(1)}(\alpha)\left[1+\frac{3(t-\alpha)}{\beta-\alpha}\right]+\frac{(t-\alpha)^{2}}{2} \cdot f^{(2)}(\alpha)\right]+ \\
\frac{(t-\alpha)^{3}}{(\beta-\alpha)^{3}} \cdot\left[f(\beta)\left[1+\frac{3(\beta-t)}{\beta-\alpha}+\frac{6(\beta-t)^{2}}{(\beta-\alpha)^{2}}\right]+(t-\beta) \cdot f^{(1)}(\beta)\left[1+\frac{3(\beta-t)}{\beta-\alpha}\right]+\frac{(\beta-t)^{2}}{2} \cdot f^{(2)}(\beta)\right] \\
f_{3}(\alpha, \beta, t)=\frac{(\beta-t)^{4}}{(\beta-\alpha)^{4}} \cdot\left[\begin{array}{l}
f(\alpha)\left[1+\frac{4(t-\alpha)}{\beta-\alpha}+\frac{10(t-\alpha)^{2}}{(\beta-\alpha)^{2}}+\frac{20(t-\alpha)^{3}}{(\beta-\alpha)^{3}}\right]+(t-\alpha) \cdot f^{(1)}(\alpha)\left[1+\frac{4(t-\alpha)}{\beta-\alpha}+\frac{10(t-\alpha)^{2}}{(\beta-\alpha)^{2}}\right]+ \\
\frac{(t-\alpha)^{2}}{2} \cdot f^{(2)}(\alpha)\left[1+\frac{4(t-\alpha)}{\beta-\alpha}\right]+\frac{(t-\alpha)^{3}}{6} \cdot f^{(3)}(\alpha)
\end{array}\right]+ \\
\frac{(t-\alpha)^{4}}{(\beta-\alpha)^{4}} \cdot\left[\begin{array}{l}
f(\beta)\left[1+\frac{4(\beta-t)}{\beta-\alpha}+\frac{10(\beta-t)^{2}}{(\beta-\alpha)^{2}}+\frac{20(\beta-t)^{3}}{(\beta-\alpha)^{3}}\right]+(t-\beta) \cdot f^{(1)}(\beta)\left[1+\frac{4(\beta-t)}{\beta-\alpha}+\frac{10(\beta-t)^{2}}{(\beta-\alpha)^{2}}\right]+ \\
\frac{(t-\beta)^{2}}{2} \cdot f^{(2)}(\beta)\left[1+\frac{4(\beta-t)}{\beta-\alpha}\right]+\frac{(t-\beta)^{3}}{6} \cdot f^{(3)}(\beta)
\end{array}\right]
\end{gathered}
$$

\section{Spline Approximation}

Consider the function defined by (33). Spline series approximations to this function, of orders $4,6,8,10$, for the interval $[0,1]$ and as defined by $(40)$, are shown in Figure 6 with the 10th order approximation visually coinciding with the function. A comparison of Figure 6 with Figure 4 shows that a spline series provides, in general, a better approximation than a dual Taylor series of the same order and with a dual Taylor series diverging more in the center of the interval of approximation. 


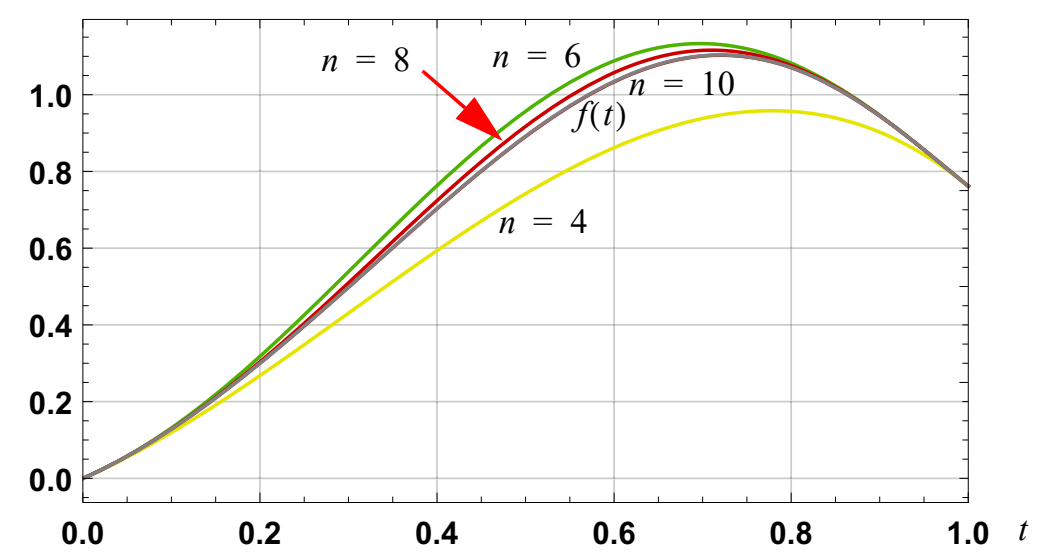

Figure 6. Graph of 4 th, 6th, 8 th and 10th order spline approximations, based on the interval [0, 1], for the function defined by (33).

\subsection{Convergence}

Consider a spline approximation, as defined by (40) or (41). A sufficient condition for convergence, i.e., $\lim _{n \rightarrow \infty} f_{n}(\alpha, \beta, t)=f(t)$, is for

$$
\lim _{n \rightarrow \infty} \frac{f_{\max }^{(n)}(\beta-\alpha)^{n}}{2^{n} \cdot n !}=0, \quad t \in[\alpha, \beta], \quad f_{\max }^{(n)}=\sup \left\{f^{(n)}(\mathrm{t}): t \in[\alpha, \beta]\right\} .
$$

The proof of this result is detailed in Appendix D.

\subsection{Spline Based Integral Approximation}

The spline approximation, as defined by (40), leads to the integral equality

$$
I(\alpha, t)=\int_{\alpha}^{t} f(\lambda) d \lambda=\sum_{k=0}^{n} c_{n, k}(t-\alpha)^{k+1}\left[f^{(k)}(\alpha)+(-1)^{k} f^{(k)}(t)\right]+R_{n}(\alpha, t)
$$

and the following spline based integral approximation

$$
I_{n}(\alpha, t)=\int_{\alpha}^{t} f_{n}(\alpha, t, \lambda) d \lambda=\sum_{k=0}^{n} c_{n, k}(t-\alpha)^{k+1}\left[f^{(k)}(\alpha)+(-1)^{k} f^{(k)}(t)\right] .
$$

In these expressions the coefficients are defined according to

$$
c_{n, k}=\frac{n+1}{k !} \sum_{i=0}^{n-k} \frac{(n+i) !}{i !} \cdot \frac{(k+i) !}{(n+k+i+2) !}
$$

A simpler expression is

$$
c_{n, k}=\frac{n !}{(n-k) !(k+1) !} \cdot \frac{(2 n+1-k) !}{2 \cdot(2 n+1) !}
$$

and this can be written, for $k \geq 1$, in the form

$$
c_{n, k}=\frac{1}{2^{k+1}(k+1) !} \cdot \prod_{i=0}^{k-1}\left[1-\frac{i}{n}\right] \cdot \prod_{i=0}^{k-1} \frac{1}{(1+(1-i) / 2 n)}, \quad k \geq 1 .
$$


The remainder is defined according to

$$
\begin{aligned}
R_{n}^{(1)}(\alpha, t)= & -\sum_{k=0}^{n} c_{n, k}(k+1)(t-\alpha)^{k}\left[f^{(k)}(\alpha)+(-1)^{k+1} \cdot \frac{n+1}{n-k+1} \cdot f^{(k)}(t)\right]+ \\
& c_{n, n}(-1)^{n+1}(t-\alpha)^{n+1} f^{(n+1)}(t)
\end{aligned}
$$

The proof of these relationships is detailed in Appendix E.

\subsubsection{Approximation in Limit}

It is the case that

$$
\lim _{n \rightarrow \infty} c_{n, k}=\frac{1}{2^{k+1} \cdot(k+1) !}, k \geq 0
$$

and it then follows, for the convergent case, that

$$
\begin{aligned}
\int_{\alpha}^{t} f(\lambda) d \lambda= & \sum_{k=0}^{n} \frac{(t-\alpha)^{k+1}}{2^{k+1}(k+1) !} \cdot\left[f^{(k)}(\alpha)+(-1)^{k} f^{(k)}(t)\right] \\
=\quad & \frac{(t-\alpha)}{2} \cdot[f(\alpha)+f(t)]+\frac{(t-\alpha)^{2}}{8} \cdot\left[f^{(1)}(\alpha)-f^{(1)}(t)\right]+\frac{(t-\alpha)^{3}}{48} \cdot\left[f^{(2)}(\alpha)-f^{(2)}(t)\right]+ \\
& \frac{(t-\alpha)^{4}}{16 \cdot 24} \cdot\left[f^{(3)}(\alpha)-f^{(3)}(t)\right]+\cdots
\end{aligned}
$$

\subsubsection{Integral Approximations of Orders Zero to Three}

The integral approximation $I_{n}(\alpha, t)$, as specified by (49), of orders zero to three are:

$$
\begin{gathered}
I_{0}(\alpha, t)=\frac{t-\alpha}{2} \cdot[f(\alpha)+f(t)] \\
I_{1}(\alpha, t)=\frac{t-\alpha}{2} \cdot[f(\alpha)+f(t)]+\frac{(t-\alpha)^{2}}{12} \cdot\left[f^{(1)}(\alpha)-f^{(1)}(t)\right] \\
I_{2}(\alpha, t)=\frac{t-\alpha}{2} \cdot[f(\alpha)+f(t)]+\frac{(t-\alpha)^{2}}{10} \cdot\left[f^{(1)}(\alpha)-f^{(1)}(t)\right]+\frac{(t-\alpha)^{3}}{120} \cdot\left[f^{(2)}(\alpha)+f^{(2)}(t)\right] \\
I_{3}(\alpha, t)=\frac{t-\alpha}{2} \cdot[f(\alpha)+f(t)]+\frac{3(t-\alpha)^{2}}{28} \cdot\left[f^{(1)}(\alpha)-f^{(1)}(t)\right]+\frac{(t-\alpha)^{3}}{84} \cdot\left[f^{(2)}(\alpha)+f^{(2)}(t)\right]+ \\
\frac{(t-\alpha)^{4}}{1680} \cdot\left[f^{(3)}(\alpha)-f^{(3)}(t)\right]
\end{gathered}
$$

\subsubsection{Remainder for Orders Zero to Three}

The remainder functions associated with a spline based integral approximation, as specified according to (53), and for orders zero to three, are defined according to

$$
\begin{gathered}
R_{0}^{(1)}(\alpha, t)=\frac{1}{2} \cdot[f(t)-f(\alpha)]-\frac{t-\alpha}{2} \cdot f^{(1)}(t) \\
R_{1}^{(1)}(\alpha, t)=\frac{1}{2} \cdot[f(t)-f(\alpha)]-\frac{(t-\alpha)}{6} \cdot\left[2 f^{(1)}(t)+f^{(1)}(\alpha)\right]+\frac{(t-\alpha)^{2}}{12} \cdot f^{(2)}(t) \\
R_{2}^{(1)}(\alpha, t)=\frac{1}{2} \cdot[f(t)-f(\alpha)]-\frac{(t-\alpha)}{10} \cdot\left[3 f^{(1)}(t)+2 f^{(1)}(\alpha)\right]+\frac{(t-\alpha)^{2}}{40} \cdot\left[3 f^{(2)}(t)-f^{(2)}(\alpha)\right]-\frac{(t-\alpha)^{3}}{120} \cdot f^{(3)}(t) \\
R_{3}^{(1)}(\alpha, t)=\frac{1}{2} \cdot[f(t)-f(\alpha)]-\frac{(t-\alpha)}{14} \cdot\left[4 f^{(1)}(t)+3 f^{(1)}(\alpha)\right]+\frac{(t-\alpha)^{2}}{28} \cdot\left[2 f^{(2)}(t)-f^{(2)}(\alpha)\right]- \\
\frac{(t-\alpha)^{3}}{420} \cdot\left[4 f^{(3)}(t)+f^{(3)}(\alpha)\right]+\frac{(t-\alpha)^{4}}{1680} \cdot f^{(4)}(t)
\end{gathered}
$$




\subsection{Explanation of Integral Approximation: Successive Area Approximation}

The integral approximation specified by (49), and the coefficients specified by (51), is best understood by considering successive approximations to the integral $\int_{\alpha}^{t} f(\lambda) d \lambda$. First, consider a zeroth order approximation as defined by

$$
\int_{\alpha}^{t} f(\lambda) d \lambda=\frac{t-\alpha}{2} \cdot[f(\alpha)+f(t)]+R_{0}(\alpha, t)=I_{0}(\alpha, t)+R_{0}(\alpha, t)
$$

and the area illustrated in Figure 7. The area is consistent with an affine approximation between the function values at the end points of the interval and equals the zeroth order integral approximation as defined by $I_{0}(\alpha, t)$. The difference between the function and an affine approximation between the values of $f(\alpha)$ and $f(t)$ defines a residual function $r_{1}$ :

$$
r_{1}(\lambda)=f(\lambda)-\left[f(\alpha)+\frac{(\lambda-\alpha)[f(t)-f(\alpha)]}{t-\alpha}\right], \lambda \in[\alpha, t] .
$$

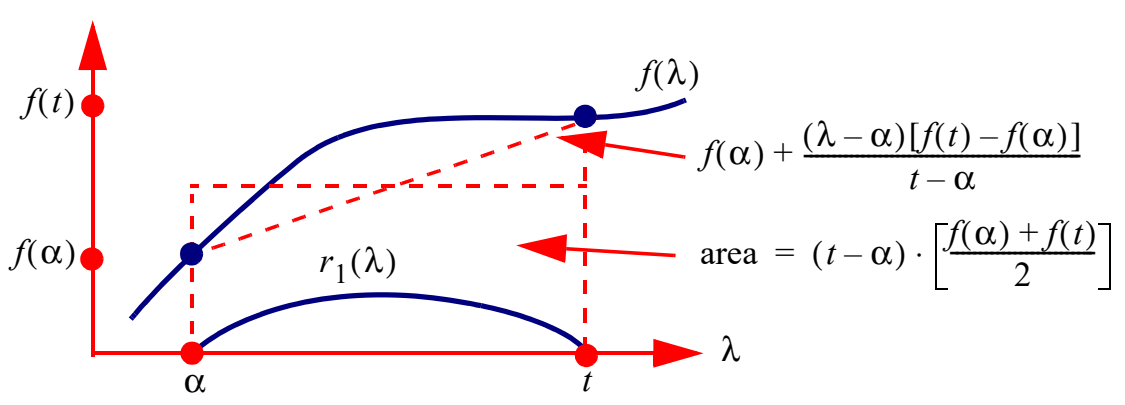

Figure 7. Illustration of the area defined by a zeroth order approximation to an integral and the residual function $r_{1}$.

Second, consider the first order approximation to the integral as defined by

$$
\int_{\alpha}^{t} f(\lambda) d \lambda=\frac{t-\alpha}{2} \cdot[f(\alpha)+f(t)]+\frac{(t-\alpha)^{2}}{12} \cdot\left[f^{(1)}(\alpha)-f^{(1)}(t)\right]+R_{1}(\alpha, t)
$$

and the area illustrated in Figure 8. The second term in this equation approximates the area under an approximation to the residual function $r_{1}$, based on linear change at both of the end points of the interval $[\alpha, t]$, and with a difference in the denominator terms of 12 versus 8 . For higher order approximations the denominator term of 12 approaches 8 .

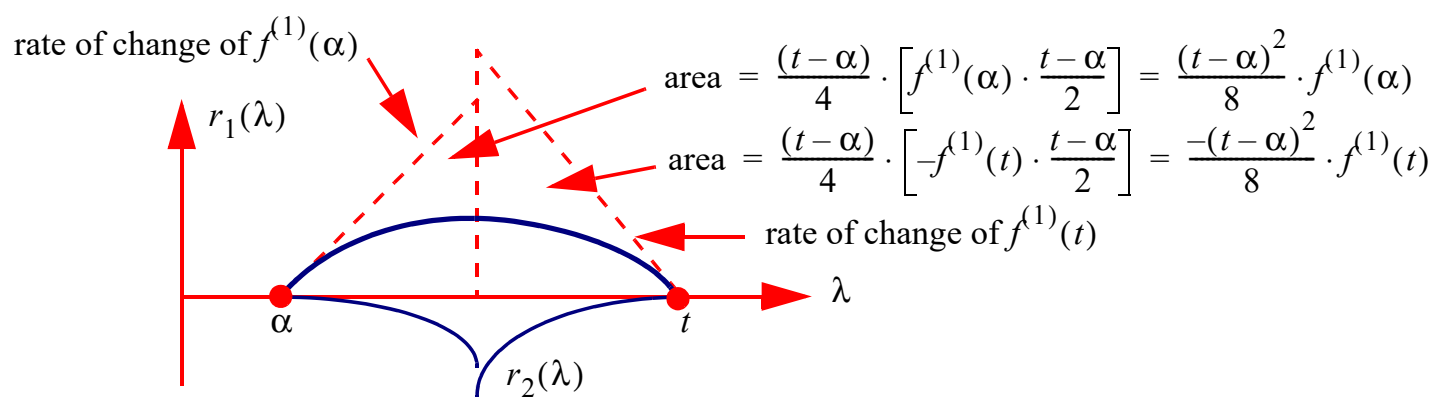

Figure 8. Illustration of the residual function $r_{1}$, the areas as defined by linear change at the points $\alpha$ and $t$ and the second residual function $r_{2}$. 
Third, consider the second order approximation to the integral as defined by

$$
\begin{aligned}
\int_{\alpha}^{t} f(\lambda) d \lambda= & \frac{t-\alpha}{2} \cdot[f(\alpha)+f(t)]+\frac{(t-\alpha)^{2}}{10} \cdot\left[f^{(1)}(\alpha)-f^{(1)}(t)\right]+ \\
& \frac{(t-\alpha)^{3}}{120} \cdot\left[f^{(2)}(\alpha)+f^{(2)}(t)\right]+R_{2}(\alpha, t)
\end{aligned}
$$

and the area illustrated in Figure 9. The third term in this expression approximates the area under a quadratic approximation to the residual function $r_{2}$, based on $f^{(2)}(\alpha)$ and $f^{(2)}(t)$, and with a different denominator term of 120 versus 48 . For higher order approximations the term of 120 approaches 48.

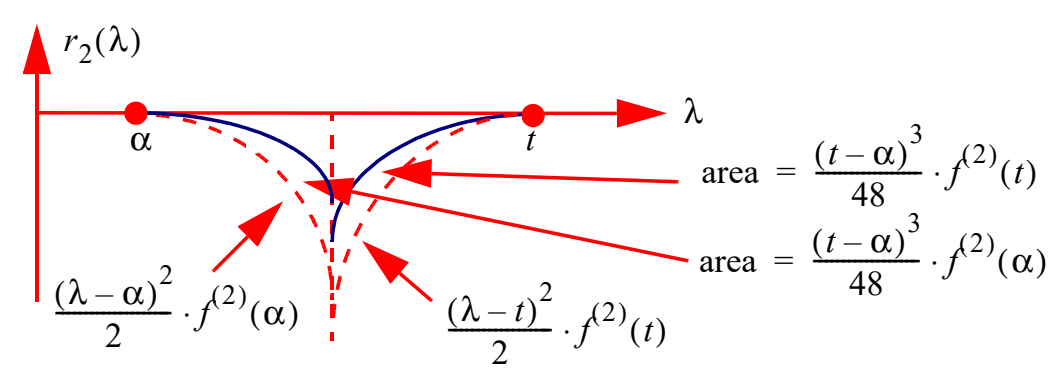

Figure 9. Illustration of the areas defined by a quadratic approximation to the residual function $r_{2}$ and based on $f^{(2)}(\alpha)$ and $f^{(2)}(t)$.

For the convergent case, the approximation to the integral, as specified by defined by (49), converges to the form specified by (55) as $n \rightarrow \infty$ and this form is consistent with the successive area approximations illustrated in Figures 7-9.

\subsection{Determining Region of Integration for a Set Error Bound}

For a positive function, a bound on the region of integration, for a set error level, can be determined by considering the area defined by the first passage time of the function to a specified level. Consider a positive function, which has a first passage time to the level $r_{B}$ at $t=t_{B}$, as illustrated in Figure 10. The following integral bound holds

$$
\int_{0}^{t_{B}} f(\lambda) d \lambda \leq r_{B} t_{B}=\varepsilon_{B},
$$

where

$$
\begin{aligned}
t_{B} & =\inf \left\{t:|f(t)|=r_{B}\right\} \\
& =\inf \left\{t: t|f(t)|=t_{B} r_{B}=\varepsilon_{B}\right\}
\end{aligned}
$$

Thus, for a bound $\varepsilon_{B}$ on the integral of a positive function $f$, a bound on the interval of integration is

$$
t_{B}=\inf \left\{t: t|f(t)|=\varepsilon_{B}\right\},
$$

i.e., the first passage time of $t|f(t)|$ to $\varepsilon_{B}$.

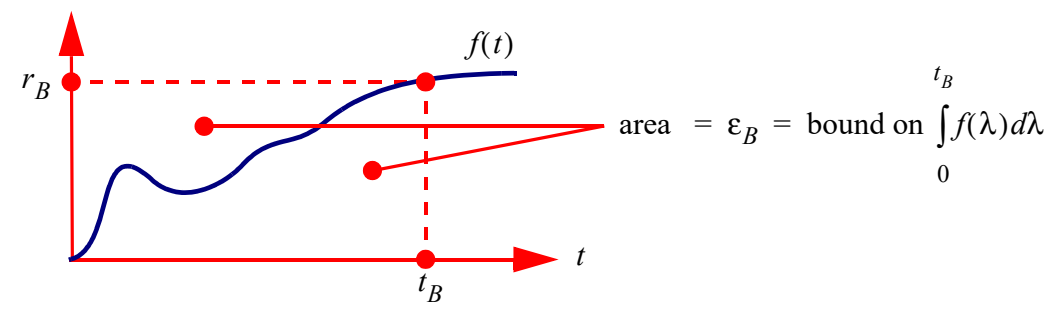

Figure 10. The area bound defined by the first passage time of a positive function to a set level. 
Consider the integral and its spline based approximation as specified by

$$
I(\alpha, t)=\int_{\alpha}^{t} f(\lambda) d \lambda=I_{n}(\alpha, t)+R_{n}(\alpha, t), \quad R_{n}(\alpha, t)=\int_{\alpha}^{t} R_{n}^{(1)}(\alpha, \lambda) d \lambda,
$$

where $R_{n}^{(1)}(\alpha, t)$ is specified by (53). As $R_{n}^{(1)}(\alpha, t)$ is known, it follows that a bound on the region of integration can be specified according to

$$
t_{B}=\inf \left\{t: t\left|R_{n}^{(1)}(t)\right|=\varepsilon_{B}\right\}
$$

and solved by standard root solving algorithms.

Example

Consider determining the region of integration, $t_{B}$, for the integral $\int_{0}^{t_{B}} \frac{\sin (t)}{1+t} d t$ and for an error bound of $\varepsilon_{B}=10^{-3}$ using the integral approximation specified by (49). The graph of $f(t)=\frac{\sin (t)}{1+t}$ is shown in Figure 11 and results are tabulated in Table 1. The specified region of integration, as expected, is conservative.

Table 1. Interval of integration for a spline based approximation and for a specified error bound of $\varepsilon_{B}=10^{-3}$.

\begin{tabular}{ccc}
\hline Order of Integral Approx. $\boldsymbol{n}$ & $\boldsymbol{t}_{\boldsymbol{B}}$ & Magnitude of Error in Integral Approx. over $\left[0, \boldsymbol{t}_{\boldsymbol{B}}\right]$ \\
\hline 1 & 0.47 & $2.4 \times 10^{-4}$ \\
2 & 0.83 & $1.9 \times 10^{-4}$ \\
3 & 1.17 & $1.5 \times 10^{-4}$ \\
4 & 1.47 & $1.3 \times 10^{-4}$ \\
6 & 1.96 & $1.0 \times 10^{-4}$ \\
8 & 2.33 & $8.2 \times 10^{-5}$ \\
10 & 2.62 & $6.9 \times 10^{-5}$ \\
12 & 2.85 & $6.0 \times 10^{-5}$ \\
\hline
\end{tabular}

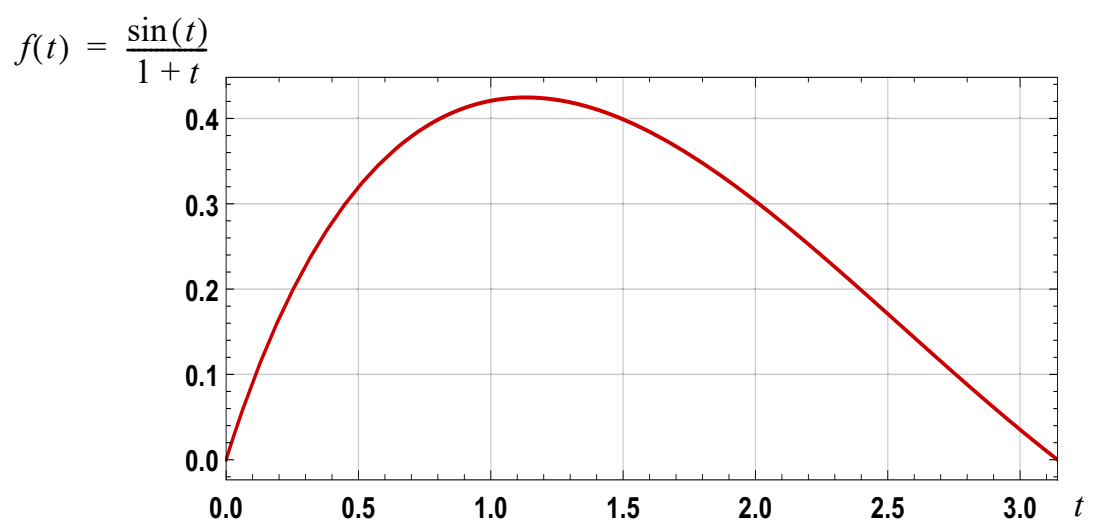

Figure 11. Graph of $f(t)=\frac{\sin (t)}{1+t}$ over the interval $[0, \pi]$.

\section{Summary and Comparison of Integral Approximations}

The function and integral approximations detailed above are summarized in Table 2 . The remainder terms associated with the integral approximations are summarized in Table 3. 
Table 2. Summary of function and integral approximations.

\begin{tabular}{|c|c|c|}
\hline & Function Approximation for $t \in[\alpha, \beta]$ & Integral Approximation: $\int_{\alpha}^{t} f(\lambda) d \lambda$ \\
\hline $\begin{array}{l}\text { Antiderivative } \\
\text { Series }\end{array}$ & & $\begin{array}{c}\sum_{k=0}^{n-1} c_{k}\left[\alpha^{k+1} f^{(k)}(\alpha)-t^{k+1} f^{(k)}(t)\right] \\
c_{k}=\frac{(-1)^{k+1}}{(k+1) !}\end{array}$ \\
\hline Taylor Series & $\sum_{k=0}^{n} \frac{(t-\alpha)^{k} f^{(k)}(\alpha)}{k !}$ & $\begin{array}{c}\sum_{k=0}^{n-1} c_{k}(t-\alpha)^{k+1} f^{(k)}(\alpha) \\
c_{k}=\frac{1}{(k+1) !}\end{array}$ \\
\hline Dual Taylor Series & $\begin{array}{l}{\left[\frac{\beta-t}{\beta-\alpha}\right]^{n+1} \sum_{k=0}^{n} \frac{(n+k) !}{n ! \cdot k !} \cdot\left[\frac{t-\alpha}{\beta-\alpha}\right]^{k} \cdot\left[\sum_{k=0}^{n} \frac{(t-\alpha)^{k} f^{(k)}(\alpha)}{k !}\right]+} \\
{\left[\frac{t-\alpha}{\beta-\alpha}\right]^{n+1} \sum_{k=0}^{n} \frac{(n+k) !}{n ! \cdot k !} \cdot\left[\frac{\beta-t}{\beta-\alpha}\right]^{k} \cdot\left[\sum_{k=0}^{n} \frac{(t-\beta)^{k} f^{(k)}(\beta)}{k !}\right]}\end{array}$ & $\begin{array}{l}\sum_{k=0}^{n} c_{n, k}(t-\alpha)^{k+1}\left[f^{(k)}(\alpha)+(-1)^{k} f^{(k)}(t)\right] \\
c_{n, k}=\frac{n+1}{k !} \cdot \sum_{u=0}^{n+1} \frac{(-1)^{u}}{u !(n+1-u) !} \\
{\left[\sum_{v=0}^{n} \frac{(n+v) !}{v !} \cdot \frac{1}{u+v+k+1}\right]}\end{array}$ \\
\hline Spline Series & $\begin{array}{c}{\left[\frac{\beta-t}{\beta-\alpha}\right]^{n+1} \cdot \sum_{k=0}^{n} \frac{(t-\alpha)^{k}}{k !} \cdot f^{k}(\alpha) \cdot\left[\sum_{i=0}^{n-k} \frac{(n+i) !}{i ! \cdot n !} \cdot \frac{(t-\alpha)^{i}}{(\beta-\alpha)^{i}}\right]+} \\
{\left[\frac{t-\alpha}{\beta-\alpha}\right]^{n+1} \cdot \sum_{k=0}^{n} \frac{(-1)^{k}(\beta-t)^{k}}{k !} \cdot f^{k}(\beta) \cdot\left[\sum_{i=0}^{n-k} \frac{(n+i) !}{i ! \cdot n !} \cdot \frac{(\beta-t)^{i}}{(\beta-\alpha)^{i}}\right]}\end{array}$ & $\begin{array}{c}\sum_{k=0}^{n} c_{n, k}(t-\alpha)^{k+1}\left[f^{(k)}(\alpha)+(-1)^{k} f^{(k)}(t)\right] \\
c_{n, k}=\frac{n !}{(n-k) !(k+1) !} \cdot \frac{(2 n+1-k) !}{2(2 n+1) !}\end{array}$ \\
\hline
\end{tabular}

Table 3. Summary of remainder terms.

\begin{tabular}{cc}
\hline & Remainder in Integral Approximation: $\int_{\alpha}^{t} f(\lambda) d \lambda$ \\
\hline Antiderivative Series & $R_{n}(\alpha, t)=\frac{(-1)^{n}}{n !} \int_{\alpha}^{t} \lambda^{n} f^{(n)}(\lambda) d \lambda$ \\
Taylor Series & $R_{n}(\alpha, t)=\int_{\alpha}^{t} \frac{(t-\lambda)^{n} f^{(n)}(\lambda)}{n !} d \lambda$ \\
Dual Taylor Series & $R_{n}^{(1)}(\alpha, t)=c_{n, 0}[f(t)-f(\alpha)]-\sum_{k=1}^{n} c_{n, k}(k+1)(t-\alpha)^{k} f^{(k)}(\alpha)+$ \\
& $\sum_{k=1}^{n}(-1)^{k+1}(t-\alpha)^{k} f^{(k)}(t)\left[(k+1) c_{n, k}-c_{n, k-1}\right]+$ \\
Spline Series & $c_{n, n}(-1)^{n+1}(t-\alpha)^{n+1} f^{(n+1)}(t)$ \\
$R_{n}^{(1)}(\alpha, t)=$ & $-\sum_{k=0}^{n} c_{n, k}(k+1)(t-\alpha)^{k}\left[f^{(k)}(\alpha)+(-1)^{k+1} \cdot \frac{n+1}{n-k+1} \cdot f^{(k)}(t)\right]+$ \\
$c_{n, n}(-1)^{n+1}(t-\alpha)^{n+1} f^{(n+1)}(t)$
\end{tabular}

Comparison of Integral Approximations

To compare the four different approximations to an integral that have been considered, and summarized in Table 2, it is useful to utilize a set of test functions. Consider a set of test functions based on a summation of Gaussian pulses

$$
f(t)=\sum_{i=1}^{m} a_{i} \exp \left[\frac{-\left(t-t_{i}\right)^{2}}{2 \sigma_{i}^{2}}\right], \sigma_{i}^{2}=\frac{t_{\mathrm{FWHM}_{i}}^{2}}{8 \ln (2)}
$$

where $m$ is an outcome of a Poisson random variable with parameter $\lambda=4$ (the zero case excluded), $a_{i}, i \in\{1, \ldots, m\}$, are independent outcomes of random variables with a normal distribution with zero mean and unit variance, $t_{i}, i \in\{1, \ldots, m\}$, are independent outcomes of random variables with a uniform distribution on the interval $[0,1]$ and $t_{\mathrm{FWHM}_{i}}^{2} i \in\{1, \ldots, m\}$, are independent outcomes of random variables with a uniform distribution on $[0,2]$. Examples of signals are shown in Figure 12. For 1000 independently generated signals, the proportion of approximations to $\int_{0}^{1} f(\lambda) d \lambda$, with a relative error of less than 0.01 , is detailed in Table 4 for the four integral approximations. The results show the clear superiority of the spline based integral approximation and simulation results for other types of signals indicate that this holds more generally. 
Table 4. Proportion of integral approximations, based on a set of 1000 test functions, with a relative error less than 0.01 .

\begin{tabular}{ccccc}
\hline Integral Approximation & $\begin{array}{c}\text { 4th Order } \\
\text { Approx. }\end{array}$ & $\begin{array}{c}\text { 6th Order } \\
\text { Approx. }\end{array}$ & $\begin{array}{c}\text { 8th Order } \\
\text { Approx. }\end{array}$ & $\begin{array}{c}\text { 10th Order } \\
\text { Approx. }\end{array}$ \\
\hline Antiderivative Series & $<0.01$ & 0.01 & 0.03 & 0.09 \\
Taylor Series & $<0.01$ & 0.01 & 0.02 & 0.08 \\
Dual Taylor Series & 0.13 & 0.34 & 0.40 & 0.48 \\
Spline Series & 0.56 & 0.71 & 0.78 & 0.84 \\
\hline
\end{tabular}

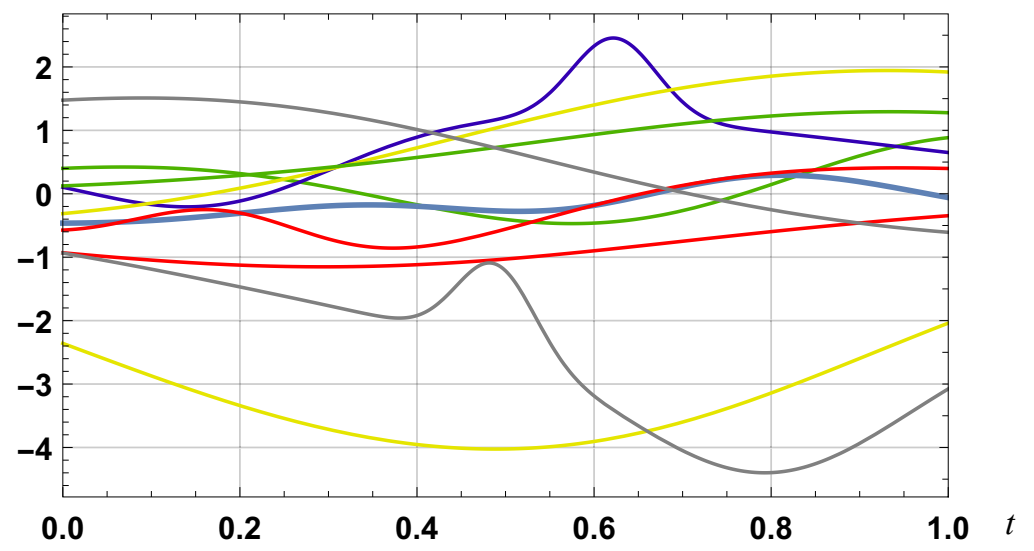

Figure 12. Examples of test functions as defined by (73).

\section{Spline Based Integral Approximation: Applications}

The integral based approximation, based on a $n$th order spline function approximation and as specified by (49), facilitates, for example, the definition of new series for standard function, new series for functions defined by integrals, and new definite integral results. In general, the series for functions have better convergence than Taylor series based approximations.

As the relative error in the evaluation of an integral, based on a spline function approximation, increases non-linearly with the region of integration, there is potential for high precision results if the value of a function defined by an integral can be established by utilizing a smaller region of integration. Several cases where this is possible are detailed.

\subsection{Exponential Function Approximation}

The spline based integral series, of order $n$, leads to the following series approximation for the exponential function

$$
\exp (t) \approx \frac{1+c_{n, 0} t+c_{n, 1} t^{2}+c_{n, 2} t^{3}+c_{n, 3} t^{4}+\cdots+c_{n, n} t^{n+1}}{1-c_{n, 0} t+c_{n, 1} t^{2}-c_{n, 2} t^{3}+c_{n, 3} t^{4}-\cdots+c_{n, n}(-1)^{n+1} t^{n+1}}
$$

where $c_{n, k}$ is specified by (51). For the case of $n \rightarrow \infty$

$$
\exp (t) \approx \frac{1+\frac{t}{2}+\frac{t^{2}}{2^{2} \cdot 2 !}+\frac{t^{3}}{2^{3} \cdot 3 !}+\frac{t^{4}}{2^{4} \cdot 4 !}+\cdots}{1-\frac{t}{2}+\frac{t^{2}}{2^{2} \cdot 2 !}-\frac{t^{3}}{2^{3} \cdot 3 !}+\frac{t^{4}}{2^{4} \cdot 4 !}-\cdots}
$$

The series converges for all $t \in R$. The proof of this result is detailed in Appendix F. 


\subsubsection{Notes}

The series defined by (74) is the same as that arising from a $(n+1)$ th order Pade approximation, e.g., [17], which can be seen by comparing the approximations for explicit orders. A fourth order approximation (fifth order 5/5 Padé approximation) is

$$
e^{t} \approx \frac{1+\frac{t}{2}+\frac{t^{2}}{9}+\frac{t^{3}}{72}+\frac{t^{4}}{1008}+\frac{t^{5}}{30240}}{1-\frac{t}{2}+\frac{t^{2}}{9}-\frac{t^{3}}{72}+\frac{t^{4}}{1008}-\frac{t^{5}}{30240}}
$$

A direct application of the series approximation as specified by (74) is the following $n$th order series approximation for the Gaussian function:

$$
\exp \left(-t^{2}\right) \approx \frac{1-c_{n, 0} t^{2}+c_{n, 2} t^{4}-c_{n, 3} t^{6}+\ldots+c_{n, n}(-1)^{n+1} t^{2 n+2}}{1+c_{n, 0} t^{2}+c_{n, 2} t^{4}+c_{n, 3} t^{6}+\ldots+c_{n, n} t^{2 n+2}}
$$

\subsubsection{Nature of Convergence}

A $n$th order Taylor series approximation for $\exp (t)$, based on the origin, leads to the well known approximation

$$
\exp (t)=\sum_{k=0}^{n} \frac{t^{k}}{k !}
$$

The relative error in the evaluation of $\exp (-t)$, based on this Taylor series, and the spline based series expansion defined by (74), is shown in Figure 13. The superiority of the spline based series is clearly evident.

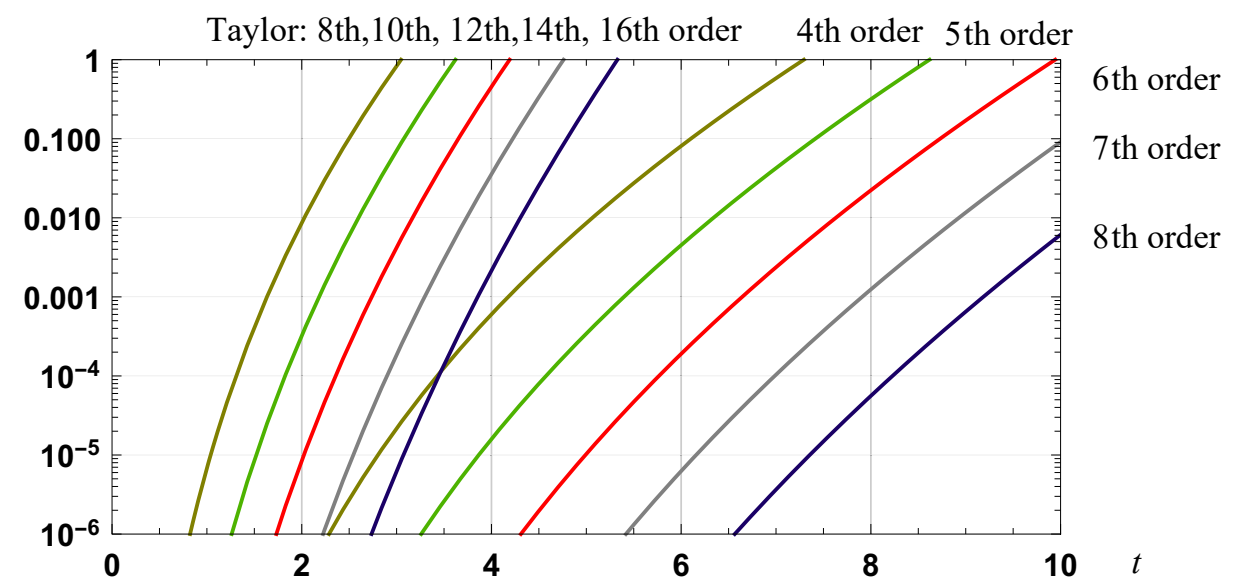

Figure 13. Graph of the relative error in approximations to exp $(-t)$ : fourth to eighth order spline based approximations along with eighth to sixteen order Taylor series approximations.

\subsubsection{High Precision Evaluation}

To establish a series with a high rate of convergence consider

$$
\begin{aligned}
e^{t} & =\left(e^{t / m}\right)^{m} \\
& \approx\left[\frac{1+c_{n, 0}(t / m)+c_{n, 1}(t / m)^{2}+c_{n, 2}(t / m)^{3}+c_{n, 3}(t / m)^{4}+\ldots+c_{n, n}(t / m)^{n+1}}{1-c_{n, 0}(t / m)+c_{n, 1}(t / m)^{2}-c_{n, 2}(t / m)^{3}+c_{n, 3}(t / m)^{4}-\ldots+c_{n, n}(-1)^{n+1}(t / m)^{n+1}}\right]^{m}
\end{aligned}
$$

High precision results can be obtained as $m$ is increased. Results that are indicative of the improvement, with increasing levels of fractional power, are detailed in Table 5 where the case of approximating $e$ is considered. 
Table 5. Relative error in evaluation of $e$.

\begin{tabular}{ccc}
\hline Order of Approx. $\boldsymbol{n}$ & Fractional Power $\boldsymbol{m}$ & Magnitude of Relative Error \\
\hline 2 & 1 & $1.0 \times 10^{-5}$ \\
& 10 & $9.9 \times 10^{-12}$ \\
& 100 & $9.9 \times 10^{-18}$ \\
& 1000 & $9.9 \times 10^{-24}$ \\
4 & 1 & $1.0 \times 10^{-10}$ \\
& 10 & $9.9 \times 10^{-21}$ \\
& 100 & $9.9 \times 10^{-31}$ \\
& 1000 & $9.9 \times 10^{-41}$ \\
& 1 & $1.7 \times 10^{-22}$ \\
& 10 & $1.7 \times 10^{-40}$ \\
& 100 & $1.7 \times 10^{-58}$ \\
& 1000 & $1.7 \times 10^{-76}$ \\
& 1 & $4.2 \times 10^{-50}$ \\
& 10 & $4.1 \times 10^{-84}$ \\
& 100 & $4.1 \times 10^{-118}$ \\
& 1000 & $4.1 \times 10^{-152}$ \\
\hline
\end{tabular}

\subsection{Natural Logarithm Approximation}

A similar approach to that used for the exponential function leads to the following $n$th order series for the natural logarithm function:

$$
\ln (t) \approx \frac{2(t-1)}{t+1} \cdot\left[1+\sum_{k=1}^{n} c_{n, k}(-1)^{k+1}(k-1) !(t-1)^{k}\left[1+\frac{(-1)^{k}}{t^{k}}\right]\right],
$$

where $c_{n, k}$ is specified by (51). The proof of this result is detailed in Appendix G.

A Taylor series, based on the point $t=1$, for the natural logarithm is

$$
\ln (t)=\sum_{k=1}^{n} \frac{(-1)^{k+1}(t-1)^{k}}{k}
$$

The relative error in this series, and the spline based series defined by (80), is detailed in Figure 14 . Simulation results indicate a region of convergence close to $0.15<t<6$ for the spline based series-proof of a definitive bound is an unsolved problem.

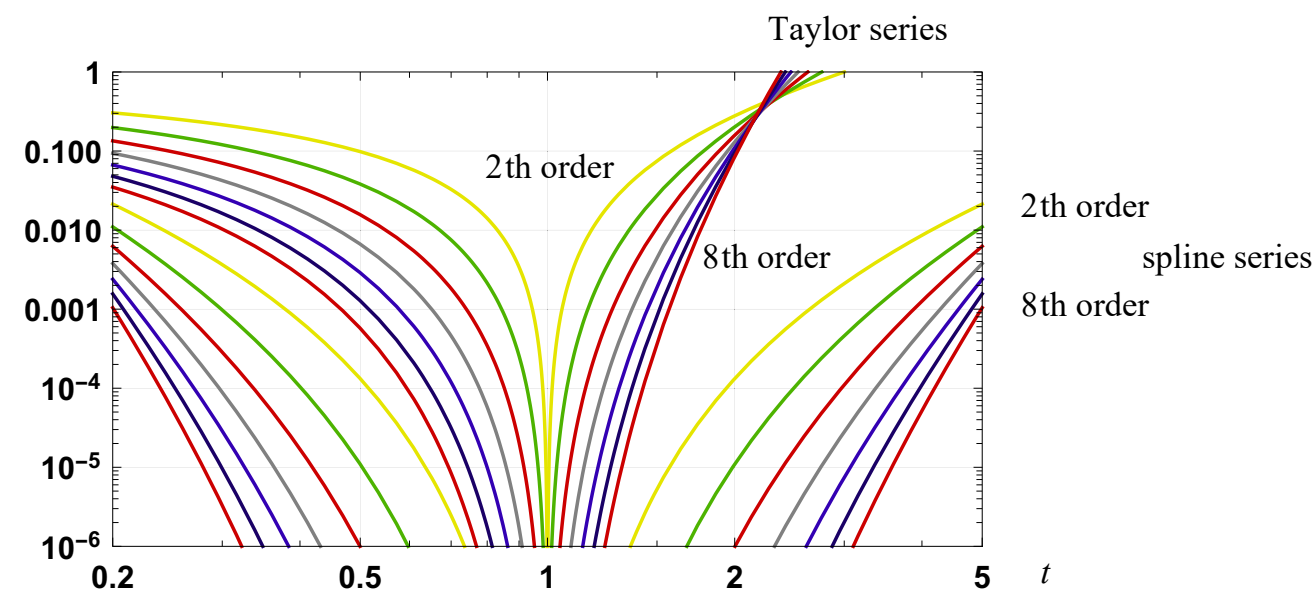

Figure 14. Graph of the relative error in approximations to $\ln (t)$ : second to eighth order Taylor series and spline based series approximation. 


\subsubsection{Example}

A fourth order approximation is

$$
\ln (t) \approx \frac{2(t-1)}{t+1}\left[1+\frac{(t-1)^{2}}{9 t}-\frac{\left(t^{2}+1\right)(t-1)^{2}}{72 t^{2}}+\frac{\left(t^{3}-1\right)(t-1)^{3}}{504 t^{3}}-\frac{\left(t^{4}+1\right)(t-1)^{4}}{5040 t^{4}}\right] .
$$

\subsubsection{High Precision Evaluation of Natural Logarithm}

As the integral of $\ln (t)$ is

$$
\int_{1}^{t} \ln (\lambda) d \lambda=t \ln (t)-t+1
$$

it follows that

$$
\int_{1}^{t^{1 / m}} \ln (\lambda) d \lambda=\frac{t^{1 / m}}{m} \ln (t)-t^{1 / m}+1 \Rightarrow \ln (t)=\frac{m}{t^{1 / m}}\left[t^{1 / m}-1\right]+\frac{m}{t^{1 / m}} \int_{1}^{t^{1 / m}} \ln (\lambda) d \lambda .
$$

Using the spline series approximation specified in (80), it follows that a $n$th order series approximation for $\ln (t)$, with higher precision and a greater range of convergence, is

$$
\ln (t) \approx \frac{2 m t^{1 / m}}{1+t^{1 / m}} \cdot\left[1-\frac{1}{t^{1 / m}}\right]+\frac{2 m}{1+t^{1 / m}} \cdot \sum_{k=1}^{n} c_{n, k}(-1)^{k+1}(k-1) !\left(t^{1 / m}-1\right)^{k+1}\left[1+\frac{(-1)^{k}}{t^{k / m}}\right]
$$

where $c_{n, k}$ is specified by (51). Results that are indicative of the improvement in precision, with increasing levels of fractional power, are detailed in Table 6.

Table 6. Relative error in evaluation of $\ln (t)$ for the case of $t=100$. For the case of $m=1$ the relative error is high for all orders of approximation.

\begin{tabular}{ccc}
\hline Order of Approx. $\boldsymbol{n}$ & Fractional Power $\boldsymbol{m}$ & Magnitude of Relative Error \\
\hline 2 & 10 & $1.1 \times 10^{-5}$ \\
& 100 & $1.1 \times 10^{-11}$ \\
4 & 1000 & $1.1 \times 10^{-17}$ \\
& 10 & $1.6 \times 10^{-8}$ \\
& 100 & $1.5 \times 10^{-18}$ \\
\multirow{2}{*}{8} & 1000 & $1.5 \times 10^{-28}$ \\
& 10 & $5.7 \times 10^{-14}$ \\
& 100 & $5.2 \times 10^{-32}$ \\
& 1000 & $5.2 \times 10^{-50}$ \\
& 10 & $1.6 \times 10^{-24}$ \\
& 100 & $1.3 \times 10^{-58}$ \\
& 1000 & $1.3 \times 10^{-92}$ \\
\hline
\end{tabular}

\subsubsection{High Precision Evaluation of $\ln (2)$}

As a second example, consider the evaluation of $\ln (2)$ which can be defined according to

$$
\ln (2)=\int_{0}^{1} \frac{1}{1+t} d t=0.6931471805 \cdots
$$


One associated series, e.g., [18], p. 15, is

$$
\ln (2)=\lim _{n \rightarrow \infty} \sum_{k=1}^{n} \frac{1}{n+k}
$$

whilst Taylor series for $\ln (x)$, based on the points at 1 and 2, yield

$$
\ln (2)=\sum_{k=1}^{\infty} \frac{(-1)^{k-1}}{k}, \quad \ln (2)=\sum_{k=1}^{\infty} \frac{1}{k \cdot 2^{k^{\prime}}}
$$

i.e.,

$$
\ln (2)=1-\frac{1}{2}+\frac{1}{3}-\frac{1}{4}+\cdots, \quad \ln (2)=\frac{1}{2}+\frac{1}{2 \cdot 4}+\frac{1}{3 \cdot 8}+\cdots .
$$

The second series arises by finding an approximation for $\ln (1)$ based on the Taylor series at the point 2 .

The relative errors in the Taylor series for orders $2,4,8,16$, respectively, are: $0.28,0.16,0.085$ and 0.044 for the first series and $0.098,0.016,5.7 \times 10^{-4}$ and $1.2 \times 10^{-6}$ for the second series. The relative errors in the series defined by (87), for orders $2,4,8,16$, are: $0.16,0.085,0.044$ and 0.022 .

A second order series specified by (85) is

$$
\ln (2) \approx 2 m \cdot \frac{2^{1 / m}-1}{2^{1 / m}+1}\left[1+\frac{\left(2^{1 / m}-1\right)^{2}}{10 \cdot 2^{1 / m}}-\frac{\left(2^{1 / m}-1\right)^{2}\left(2^{2 / m}+1\right)}{120 \cdot 2^{2 / m}}\right]
$$

and yields an approximation with relative errors, respectively, of $1.3 \times 10^{-4}, 1.3 \times 10^{-10}, 1.3 \times 10^{-16}$ and $1.3 \times 10^{-22}$ for the cases of $m=1,10,100,1000$.

\subsection{Evaluation of Numbers to Fractional Powers}

Consider determining $x_{o}^{1 / h}$ for the case of $h \in\{1,2, \ldots\}$. With an initial approximation to $x_{o}^{1 / h}$ of $z_{0}$, the following integral is the basis for an iterative algorithm:

$$
I_{0}=\int_{z_{0}^{h}}^{x_{0}} \frac{1}{h t^{1-1 / h}} d t=\left.t^{1 / h}\right|_{z_{0}^{h}} ^{x_{0}}=x_{o}^{1 / h}-z_{0} \Rightarrow x_{o}^{1 / h}=z_{0}+I_{0} .
$$

An approximation to $I_{0}$ yields an approximation for $x_{0}^{1 / h}$ which is denoted $z_{1}$ where

$$
x_{o}^{1 / h} \approx z_{1}=z_{0}+\text { approximation to } I_{0} .
$$

Replacing $z_{0}^{h}$ by $z_{1}^{h}$ in (91) is the first step in an iterative algorithm to establish an accurate approximation to $x_{o}^{1 / h}$. The requirement for such an algorithm is a suitable approximation for the integral defined by $I_{0}$ and a $n$th order spline integral approximation is useful.

\subsubsection{Iterative Algorithm}

An iterative algorithm for determining an approximation to $x_{0}^{1 / h}$, and based on a $n$th order spline integral approximation, is:

$$
x_{o}^{1 / h} \approx \frac{z_{i}+u_{i+1}}{1-v_{i+1}}, z_{i}=\frac{z_{i-1}+u_{i}}{1-v_{i}}
$$


where an initial number, less than $x_{0}^{1 / h}$, of $z_{0}$ is chosen and

$$
\begin{aligned}
& u_{i+1}=\sum_{k=0}^{n} c_{n, k}(-1)^{k+1}\left(x_{0}-z_{i}^{h}\right)^{k+1} \cdot \prod_{j=0}^{k}\left(j-\frac{1}{h}\right) \cdot\left[\frac{1}{z_{i}^{k h+h-1}}\right], \\
& v_{i+1}=-\sum_{k=0}^{n} c_{n, k}\left(x_{0}-z_{i}^{h}\right)^{k+1} \cdot \prod_{j=0}^{k}\left(j-\frac{1}{h}\right) \cdot\left[\frac{1}{x_{0}^{k+1}}\right]
\end{aligned}
$$

Here $c_{n, k}$ is specified by (51). The proof of this algorithm is detailed in Appendix H.

\subsubsection{Example and Results}

For a 3rd order spline integral approximation, the algorithm is based on

$$
\begin{aligned}
u_{i+1}= & \left(x_{0}-z_{i}^{h}\right) \cdot \frac{1}{2 h z_{i}^{h-1}}-\left(x_{0}-z_{i}^{h}\right)^{2} \cdot \frac{3(h-1)}{28 h^{2} z_{i}^{2 h-1}}+\left(x_{0}-z_{i}^{h}\right)^{3} \cdot \frac{(h-1)(2 h-1)}{84 h^{3} z_{i}^{3 h-1}}- \\
& \left(x_{0}-z_{i}^{h}\right)^{4} \cdot \frac{(h-1)(2 h-1)(3 h-1)}{1680 h^{4} z_{i}^{4 h-1}} \\
v_{i+1}= & \left(x_{0}-z_{i}^{h}\right) \cdot \frac{1}{2 h x_{0}}+\left(x_{0}-z_{i}^{h}\right)^{2} \cdot \frac{3(h-1)}{28 h^{2} x_{0}^{2}}+\left(x_{0}-z_{i}^{h}\right)^{3} \cdot \frac{(h-1)(2 h-1)}{84 h^{3} x_{0}^{3}}+ \\
& \left(x_{0}-z_{i}^{h}\right)^{4} \cdot \frac{(h-1)(2 h-1)(3 h-1)}{1680 h^{4} x_{0}^{4}}
\end{aligned}
$$

Indicative results for the efficacy of the iterative algorithm for evaluation of a rational number to a fractional power, based on third and sixth order spline approximations, are detailed in Table 7.

Table 7. Relative error in evaluation of $x_{o}^{1 / h}$ for the case of $x_{o}=137, h=6$ and an initial estimate for $x_{o}^{1 / h}$ of $9 / 5.137^{1 / 6}=2.27049261 \ldots$.

\begin{tabular}{ccc}
\hline Spline Approximation Order: $\boldsymbol{n}$ & Iteration order & Magnitude of Relative Error \\
\hline 3 & 1 & $5.1 \times 10^{-3}$ \\
& 2 & $4.2 \times 10^{-18}$ \\
& 3 & $7.2 \times 10^{-154}$ \\
6 & 1 & $6.7 \times 10^{-4}$ \\
& 2 & $2.0 \times 10^{-42}$ \\
& 3 & $3.2 \times 10^{-620}$ \\
\hline
\end{tabular}

\subsection{Arc-Cosine, Arc-Sine and Arc-Tangent Function Approximation}

Given the coordinate $(x, y)$ of a point on the first quadrant of the unit circle, the corresponding angle $\theta$, as defined by $\theta=\operatorname{acos}(x), \theta=\operatorname{asin}(y)$ and $\theta=\operatorname{atan}(y / x)$, can be determined from the following integral which is associated with the angle $\theta / 2^{i}, i \in\{1,2, \ldots\}$ :

$$
\theta=2^{i} \cdot \int_{0}^{\frac{g_{i}(x)}{\sqrt{1+g_{i}^{2}(x)}}} \frac{1}{\sqrt{1-\lambda^{2}}} d \lambda, i \in\{1,2, \ldots\} .
$$

This result is proved in Appendix I. To determine an approximation for $\theta$, and, hence, $\operatorname{acos}(x), \operatorname{asin}(y)$ and $\operatorname{atan}(\mathrm{y} / x)$, first, $\mathrm{g}_{i}(x)$ needs to be specified. Second, an approximation to the integral needs to be specified and the spline based integral approximation is efficacious. 
6.4.1. Algorithm for Determining $g_{i}(x)$

An algorithm for determining $g_{i}(x), i \in\{1,2, \ldots\}$, is:

$$
\begin{gathered}
g_{i}(x)=\frac{s_{i-1}}{1+c_{i-1}}, \quad s_{i}=\frac{1}{\sqrt{2}} \cdot \sqrt{1-c_{i-1}}, \quad c_{i}=\frac{1}{\sqrt{2}} \cdot \sqrt{1+c_{i-1}}, \\
s_{0}=\sin (\theta)=\sqrt{1-x^{2}}, \quad c_{0}=\cos (\theta)=x
\end{gathered}
$$

Here:

$$
g_{i}(x)=\tan \left(\theta / 2^{i}\right), \quad s_{i}=\sin \left(\theta / 2^{i}\right), \quad c_{i}=\cos \left(\theta / 2^{i}\right) .
$$

A direct definition for $g_{i}(x)$ is

$$
g_{0}(x)=\frac{\sqrt{1-x^{2}}}{x}, \quad g_{1}(x)=\frac{\sqrt{1-x^{2}}}{1+x}, \quad g_{i}(x)=\frac{\sqrt{2-\sqrt{d_{i-2}}}}{2+\sqrt{d_{i-1}}}, i \geq 2
$$

where an algorithm for determining $d_{i}$ is:

$$
d_{0}=4 x^{2}, \quad d_{1}=2(1+x), \quad d_{i}=2+\sqrt{d_{i-1}}, \quad i \geq 2 .
$$

The proof of these results is detailed in Appendix I.

As an example, the third and fourth order functions $g_{3}(x)$ and $g_{4}(x)$ are:

$$
g_{3}(x)=\frac{\sqrt{2-\sqrt{2} \sqrt{1+x}}}{2+\sqrt{2+\sqrt{2} \sqrt{1+x}}}, \quad g_{4}(x)=\frac{\sqrt{2-\sqrt{2+\sqrt{2} \sqrt{1+x}}}}{2+\sqrt{2+\sqrt{2+\sqrt{2} \sqrt{1+x}}}} .
$$

\subsubsection{Spline Based Integral Approximation}

The integral defined by (97) can be approximated by using a $n$th order spline integral approximation as defined by (49) and (51). For the case of $(x, y)$ specified with $y=\sqrt{1-x^{2}}$, approximations to $\theta=\operatorname{atan}(y / x), \theta=\operatorname{acos}(x)$ and $\theta=\operatorname{asin}(y)$ can be determined. Based on a $n$th order spline integral approximation, the approximation is

$$
\theta \approx 2^{i} \sum_{k=0}^{n} c_{n, k} \cdot\left[\frac{g_{i}(x)}{\sqrt{1+g_{i}^{2}(x)}}\right]^{k+1} \cdot\left[p[k, 0]+(-1)^{k} p\left[k, \frac{g_{i}(x)}{\sqrt{1+g_{i}^{2}(x)}}\right]\left[1+g_{i}^{2}(x)\right]^{(2 k+1) / 2}\right],
$$

where $c_{n, k}$ is specified by (51) and

$$
\begin{aligned}
& p(0, t)=1 \\
& p(k, t)=\left(1-t^{2}\right) \frac{d}{d t} p(k-1, t)+(2 k-1) t p(k, t)
\end{aligned}
$$

As an example

$$
p(6, t)=45\left(5+90 t^{2}+120 t^{4}+16 t^{6}\right)
$$

These results arise from the spline based integral approximation as specified by (49) and (51) and by noting that

$$
f(t)=\frac{1}{\left[1-t^{2}\right]^{1 / 2}} \Rightarrow f^{(k)}(t)=\frac{p(k, t)}{\left[1-t^{2}\right]^{(2 k+1) / 2}}, k \in\{0,1, \ldots\},
$$


where the algorithm for determining the numerator polynomial $p(k, t)$ is specified by $(104)$. Substitution of this result into (49) yields

$$
\theta \approx 2^{i} \sum_{k=0}^{n} c_{n, k}\left[\frac{g_{i}(x)}{\sqrt{1+g_{i}^{2}(x)}}\right]^{k+1}\left[p[k, 0]+\frac{(-1)^{k} p\left[k, \frac{g_{i}(x)}{\sqrt{1+g_{i}^{2}(x)}}\right]}{\left[1-\frac{g_{i}^{2}(x, y)}{1+g_{i}^{2}(x, y)}\right]^{(2 k+1) / 2}}\right] .
$$

Simplification yields the result stated in (103).

\subsubsection{Example and Results}

With $p[0, t]=1, p[1, t]=t, p[2, t]=1+2 t^{2}, p(3, t)=3 t\left(3+2 t^{2}\right)$ it follows that a third order spline based approximation is

$$
\theta \approx 2^{i}\left[\begin{array}{l}
\frac{1}{2} \cdot \frac{g_{i}(x)}{\sqrt{1+g_{i}^{2}(x)}} \cdot\left[1+\left[1+g_{i}^{2}(x)\right]^{1 / 2}\right]-\frac{3}{28} g_{i}^{3}(x)+ \\
\frac{1}{84} \cdot \frac{g_{i}^{3}(x)}{\left[1+g_{i}^{2}(x)\right]^{3 / 2}} \cdot\left[1+\left[1+3 g_{i}^{2}(x)\right] \cdot\left[1+g_{i}^{2}(x)\right]^{3 / 2}\right]-\frac{1}{560} \cdot g_{i}^{5}(x) \cdot\left[3+5 g_{i}^{2}(x)\right]
\end{array}\right]
$$

where, for the case of $i=4, g_{4}(x)$ is specified in (102). The use of $g_{4}(x)$ in (108), for the case of $x=1 / \sqrt{2}$, yields an approximation to atan $(1)=\pi / 4$ and, hence, $\pi$ with a relative error of $1.5 \times 10^{-14}$. Weisstein [19] provides a good overview of approaches for calculating $\pi$. Further, and indicative, results are tabulated in Table 8. High precision results can be established, for relatively low order spline based integral approximations, by using a high order of angle subdivision.

Table 8. Relative error in approximation of $\pi$ based on an approximation to atan(1).

\begin{tabular}{ccc}
\hline Order of Spline Approx. $\boldsymbol{n}$ & Order of Angle Sub-division $\boldsymbol{i}$ & Magnitude of Relative Error \\
\hline 1 & 1 & $4.4 \times 10^{-3}$ \\
& 2 & $2.1 \times 10^{-5}$ \\
4 & 10 & $7.3 \times 10^{-8}$ \\
& 100 & $4.3 \times 10^{-15}$ \\
& 1 & $1.8 \times 10^{-123}$ \\
& 2 & $1.7 \times 10^{-5}$ \\
& 4 & $1.6 \times 10^{-7}$ \\
& 10 & $3.2 \times 10^{-11}$ \\
& 100 & $4.5 \times 10^{-22}$ \\
& 1 & $1.3 \times 10^{-184}$ \\
& 2 & $3.6 \times 10^{-8}$ \\
& 4 & $1.2 \times 10^{-11}$ \\
& 10 & $7.5 \times 10^{-18}$ \\
& 100 & $6.3 \times 10^{-36}$ \\
& 1 & $7.4 \times 10^{-307}$ \\
& 2 & $2.5 \times 10^{-13}$ \\
& 4 & $1.3 \times 10^{-19}$ \\
& 10 & $6.1 \times 10^{-31}$ \\
& 100 & $1.7 \times 10^{-63}$ \\
\end{tabular}

\subsection{Series for Integral Defined Functions: The Fresnel Sine Integral}

The spline based integral approximation, as specified by (49), can be used to define series approximations for integral defined functions. As an example, consider the Fresnel sine integral $\int_{0}^{t} \sin \left(\lambda^{2}\right) d \lambda$. To establish a spline based approximation for this integral the $k$ th derivative of 
$f(t)=\sin \left(t^{2}\right)$ is required. This can be specified by using the quadrature signal $f_{q}(t)=\cos \left(t^{2}\right)$ and is

$$
f^{(k)}(t)=p_{k}(t) f(t)+q_{k}(t) f_{q}(t), \quad k \in\{0,1, \ldots\},
$$

where

$$
\begin{aligned}
& p_{0}(t)=1, \quad q_{0}(t)=0 \\
& p_{1}(t)=0, \quad q_{1}(t)=2 t \\
& p_{k}(t)=p_{k-1}^{(1)}(t)-2 t q_{k-1}(t), \quad q_{k}(t)=q_{k-1}^{(1)}(t)+2 t p_{k-1}(t), k \geq 2
\end{aligned}
$$

It then follows that a spline based approximation to the Fresnel sine integral is:

$$
\begin{aligned}
\int_{0}^{t} \sin \left(\lambda^{2}\right) d \lambda \approx & \sum_{k=2,6,10, \ldots . .}^{n} c_{n, k} t^{k+1} q_{k}(0)+ \\
& \sin \left(t^{2}\right) \sum_{k=0}^{n} c_{n, k}(-1)^{k} t^{k+1} p_{k}(t)+\cos \left(t^{2}\right) \sum_{k=0}^{n} c_{n, k}(-1)^{k} t^{k+1} q_{k}(t)
\end{aligned}
$$

where $c_{n, k}$ is specified by (51). A sixth order approximation is

$$
\int_{0}^{t} \sin \left(\lambda^{2}\right) d \lambda \approx \frac{5 t^{3}}{156}-\frac{t^{7}}{144144}+\sin \left(t^{2}\right)\left[\frac{t}{2}-\frac{409 t^{5}}{8580}+\frac{997 t^{9}}{1081080}-\frac{t^{13}}{270270}\right]+\cos \left(t^{2}\right)\left[-\frac{31 t^{3}}{156}+\frac{56511^{7}}{720720}-\frac{411^{11}}{540540}\right]
$$

The error in a sixth order approximation to the integral is illustrated in Figure 15. The relative error is significantly less than for a standard Taylor series approximation defined, e.g., [20,21], according to

$$
\int_{0}^{t} \sin \left(\lambda^{2}\right) d \lambda \approx \sum_{k=0}^{\infty} \frac{(-1)^{k} t^{4 k+3}}{(4 k+3) \cdot(2 k+1) !}=\frac{t^{3}}{3}-\frac{t^{7}}{42}+\frac{t^{11}}{1320}-\ldots
$$

For integration over the interval $[0,1.5]$, the true integral is $0.778238 \ldots$ and a 6 th order spline approximation yields a relative error of $-1.4 \times 10^{-5}$.

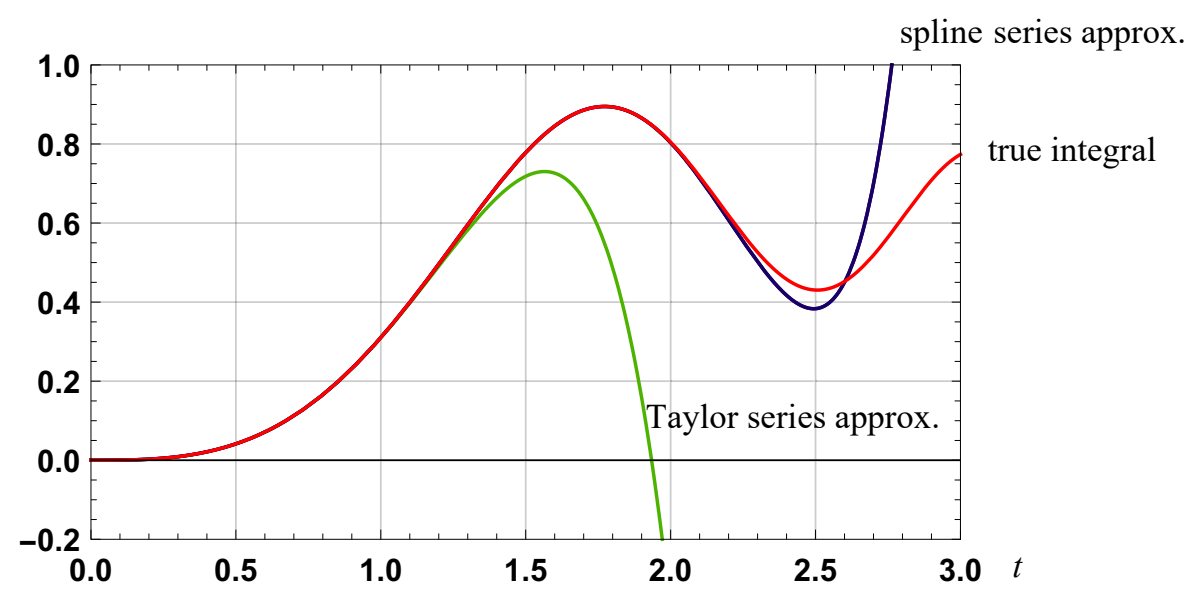

Figure 15. Graph of approximations (order 6) to the integral of $\sin \left(t^{2}\right)$.

\subsection{Definite Integrals}

The following examples illustrate the ability of spline based integral approximation to define new series for definite integrals. 


\subsubsection{Example 1}

Consider the approximation

$$
\int_{0}^{t} \frac{\sin (\lambda)}{1+\lambda} d \lambda \approx \sum_{k=0}^{n} c_{n, k} t^{k+1} \cdot \sum_{i=0}^{k} \frac{(-1)^{i} k !}{(k-i) !} \cdot\left[\sin \left[\frac{(k-i) \pi}{2}\right]+\sin \left[t+\frac{(k-i) \pi}{2}\right] \cdot \frac{(-1)^{k}}{(1+t)^{i+1}}\right]
$$

arising from (49), with $c_{n, k}$ as specified by (51), and based on the result

$$
\frac{\mathrm{d}^{k}}{\mathrm{~d} t^{k}}[u(t) v(t)]=\sum_{i=0}^{k} \frac{k !}{(k-i) ! i !} \cdot u^{(k-i)} v^{(i)}(t),
$$

where $u(t)=\sin (t), v(t)=\frac{1}{1+t}, u^{(i)}(t)=\sin \left[t+\frac{i \pi}{2}\right]$ and $v^{(i)}(t)=\frac{(-1)^{i} i !}{(1+t)^{i+1}}$.

From (114), the following definite integral approximation

$$
\int_{0}^{\pi} \frac{\sin (\lambda)}{1+\lambda} d \lambda \approx \sum_{k=0}^{n} c_{n, k} \pi^{k+1} \cdot\left[\sum_{i=0}^{k} \frac{(-1)^{i} k !}{(k-i) !} \cdot \sin \left[\frac{(k-i) \pi}{2}\right]\left[1+\frac{(-1)^{k+1}}{(1+\pi)^{i+1}}\right]\right]
$$

is valid. An alternative form is

$$
\int_{0}^{\pi} \frac{\sin (\lambda)}{1+\lambda} d \lambda \approx \frac{\pi^{2}}{(1+\pi)^{n}} \cdot \sum_{v=0}^{2 n-1} d_{n, v} \pi^{v}
$$

where

$$
\begin{aligned}
& d_{n, v}=\frac{p^{(v)}(t)}{v !} \mid t \rightarrow 0 \\
& p(t)=\sum_{k=0}^{n} c_{n, k} t^{k-1} \cdot\left[\sum_{i=0}^{k} \frac{(-1)^{i} k !}{(k-i) !} \cdot \sin \left[\frac{(k-i) \pi}{2}\right] \cdot\left[(1+t)^{n}+(-1)^{k+1}(1+t)^{n-i-1}\right]\right]
\end{aligned}
$$

A sixth order approximation is

$$
\int_{0}^{\pi} \frac{\sin (\lambda)}{1+\lambda} d \lambda \approx \frac{\pi^{2}}{(1+\pi)^{6}} \cdot\left[\begin{array}{r}
\frac{3}{13}+\frac{33 \pi}{26}+\frac{4865 \pi^{2}}{1716}+\frac{11105 \pi^{3}}{3432}+\frac{5669 \pi^{4}}{2970}+\frac{145651 \pi^{5}}{308880}-\frac{2257 \pi^{6}}{332640}- \\
\frac{29977 \pi^{7}}{2882880}+\frac{157 \pi^{8}}{154440}-\frac{1333 \pi^{9}}{4324320}+\frac{101 \pi^{10}}{864864}-\frac{101 \pi^{11}}{2882880}
\end{array}\right]
$$

The true value of the integral is $0.84381081 \ldots$ and the relative errors in the series approximation for $n=4,8,16,64,128,256$, respectively, are $0.035,3.3 \times 10^{-3}, 3.9 \times 10^{-5}, 3.2 \times 10^{-16}, 9.2 \times 10^{-31}$ and $1.1 \times 10^{-59}$.

\subsubsection{Example 2: Catalan's Constant}

Consider the Catalan constant $G$ which is defined by the series

$$
G=\sum_{k=0}^{\infty} \frac{(-1)^{k}}{(2 k+1)^{2}}=0.9159655941
$$

and, equivalently, by the integral, e.g., [18], pp. 56-57,

$$
G=2 \int_{0}^{\pi / 4} \ln [2 \cos (t)] d t .
$$


A spline based approximation for Catalan's constant, based on this integral, is

$$
G \approx 2 \sum_{k=0}^{n} c_{n, k} \cdot \frac{\pi^{k+1}}{4^{k+1}} \cdot\left[f^{(k)}(0)+(-1)^{k} f^{(k)}(\pi / 4)\right],
$$

where $c_{n, k}$ is defined by (51) and

$$
f(t)=\ln [2 \cos (t)], \quad f^{(k)}(t)=\frac{N(k, t)}{\cos (t)^{k}}, \quad k \in\{1,2, \ldots\} .
$$

Here:

$$
\begin{aligned}
& N(1, t)=-\sin (t), \\
& N(k, t)=\frac{\mathrm{d}}{\mathrm{d} t} N(k-1, t) \cdot \cos (t)+(k-1) N(k-1, t) \sin (t), t \geq 2
\end{aligned}
$$

A sixth order approximation is

$$
G \approx \frac{3 \pi}{8} \cdot \ln (2)+\frac{3 \pi^{2}}{208}-\frac{5 \pi^{3}}{3328}+\frac{5 \pi^{4}}{109824}-\frac{9 \pi^{5}}{2928640}+\frac{\pi^{6}}{7907328}-\frac{\pi^{7}}{268369920}
$$

and has a relative error of $1.7 \times 10^{-8}$. A sixth order series approximation, as specified by (120), yields an approximation with a relative error of $-2.7 \times 10^{-3}$.

\subsection{Use of Sub-Division of Integration Interval}

The region of convergence for a spline based integral approximation can be extended by demarcating the region of integration into sub-intervals and by using a change of variable. Consider the integral $\int_{\alpha}^{t} f(\lambda) d \lambda$ which can be written as

$$
I=\int_{\alpha}^{\alpha+\Delta}\left[\sum_{i=0}^{m-1} f(\lambda+i \Delta)\right] d \lambda, \quad \Delta=\frac{t-\alpha}{m}
$$

and can be approximated, consistent with (49) and (51), according to

$$
I \approx \sum_{k=0}^{n} c_{n, k} \Delta^{k+1}\left[g^{(k)}(\alpha)+(-1)^{k} g^{(k)}(\Delta)\right]
$$

where

$$
g(t)=\sum_{i=0}^{m-1} f(t+i \Delta), \quad t \in[\alpha, \alpha+\Delta]
$$

\subsubsection{Example}

Consider the integral

$$
I=\int_{0}^{t} \frac{\sin (\lambda)}{1+\lambda} d \lambda=\int_{0}^{t / m}\left[\sum_{q=0}^{m-1} \frac{\sin (\lambda+q \Delta)}{1+\lambda+q \Delta}\right] d \lambda, \quad \Delta=t / m,
$$

which has the approximation detailed in (114) and where the demarcation of the interval $[0, t]$ into $m$ sub-intervals of measure $\Delta=t / m$ has been used. It then follows that

$$
I \approx \sum_{k=0}^{n} c_{n, k} \cdot \frac{t^{k+1}}{m^{k+1}} \cdot\left[g^{(k)}(0)+(-1)^{k} g^{(k)}(t / m)\right],
$$


where

$$
g(t)=\sum_{q=0}^{m-1} \frac{\sin (t+q \Delta)}{1+t+q \Delta}
$$

and, based on (115),

$$
g^{(k)}(t)=\sum_{q=0}^{m-1}\left[\sum_{i=0}^{k} \frac{k !}{(k-i) !} \cdot \sin \left[t+q \Delta+\frac{(k-i) \pi}{2}\right] \cdot \frac{(-1)^{i}}{(1+t+q \Delta)^{i+1}}\right] .
$$

For the case integration over the interval $[0, \pi]$, the use of four subdivision intervals yields, for the case of spline based integral approximations of orders $n=4,8,16,64,128,256$, relative errors, respectively, with magnitudes of $1.0 \times 10^{-6}, 4.3 \times 10^{-11}, 9.8 \times 10^{-20}, 4.5 \times 10^{-71}, 2.7 \times 10^{-139}$ and $1.4 \times 10^{-275}$. Such results indicate the significant improvement in convergence when compared with the non-subdivision case as detailed in Section 6.6.1.

For the case integration over the interval $[0,100 \pi]$, the use of 256 subdivision intervals yields, for the case of spline based integral approximations of orders $n=4,8,16,64,128,256$, relative errors, respectively, with magnitudes of $5.3 \times 10^{-5}, 3.3 \times 10^{-8}, 1.6 \times 10^{-14}, 7.5 \times 10^{-52}, 2.1 \times 10^{-101}$ and $2.3 \times 10^{-200}$. The true integral is $0.618276689 \ldots$.

\subsubsection{Example: Electric Field Generated by a Ring of Charge}

A ring in free space defined by

$$
\left\{\left(x_{1}, y_{1}, z_{1}\right): x_{1}^{2}+y_{1}^{2}=a^{2}, z_{1}=b\right\}
$$

which has a free charge density of $\rho_{f}$ on it, generates an electric field at a point $(x, y, z)$ away from the ring of

$$
E(x, y, z)=\int_{0}^{2 \pi} \frac{a \rho_{f}}{4 \pi \varepsilon_{0}} \cdot \frac{[x-a \cdot \cos (t)] i+[y-a \cdot \sin (t)] j+[z-b] k}{\left[x^{2}+y^{2}+z^{2}+a^{2}-2 a x \cos (t)-2 a y \sin (t)-2 b z\right]^{3 / 2}} d t,
$$

where $\varepsilon_{o}$ is the permittivity of free space. As an example, consider the $x$ component of the electric field which is defined by the integral

$$
E_{X}(x, y, z)=\frac{a \rho_{f}}{4 \pi \varepsilon_{o}} \cdot \int_{0}^{2 \pi} \frac{[x-a \cdot \cos (t)]}{\left[r_{o}^{2}-a_{o} \cos (t-\phi)\right]^{3 / 2}} d t=\frac{a \rho_{f}}{4 \pi \varepsilon_{o}} \cdot \int_{0}^{2 \pi} f(t) d t,
$$

where

$$
\begin{aligned}
f(t) & =\frac{x-a \cdot \cos (t)}{\left[r_{o}^{2}-a_{0} \cos (t-\phi)\right]^{3 / 2}}, \\
r_{o}^{2} & =x^{2}+y^{2}+z^{2}+a^{2}-2 b z, \quad a_{o}=2 a \sqrt{x^{2}+y^{2}}, \quad \phi=\operatorname{atan}(y / x) .
\end{aligned}
$$

Using (127), and demarcation of $[0,2 \pi]$ into $m$ sub-intervals of measure $\Delta=2 \pi / m$, this component of the electric field can be approximated according to

$$
E_{X}(x, y, z) \approx \frac{a \rho_{f}}{4 \pi \varepsilon_{0}} \cdot \sum_{k=0}^{n} c_{n, k} \Delta^{k+1}\left[g^{(k)}(0)+(-1)^{k} g^{(k)}(\Delta)\right]
$$

where

$$
g(t)=\sum_{i=0}^{m-1} f(t+i \Delta), \quad g^{(k)}(t)=\sum_{i=0}^{m-1} f^{(k)}(t+i \Delta)
$$


and

$$
f^{(k)}(t) \frac{N(k, t)}{\left[r_{o}^{2}-a_{0} \cos (t+\phi)\right]^{(2 k+3) / 2}}, \quad k \in\{0,1, \ldots\} .
$$

The algorithm for determining $N(k, t)$ is

$$
\begin{aligned}
& N(0, t)=x-a \cdot \cos (t), \\
& N(k, t)=\frac{\mathrm{d}}{\mathrm{d} t} N(k-1, t) \cdot\left[r_{0}^{2}-a_{0} \cos (t-\phi)\right]-\frac{(2 k+1)}{2} N(k-1, t) a_{0} \sin (t-\phi) .
\end{aligned}
$$

For example (simplification via Mathematica):

$$
\begin{aligned}
f^{(4)}(t)= & \frac{-a \cos (t)}{\left[r_{0}^{2}-a_{0} \cos (t-\phi)\right]^{3 / 2}}+\frac{3 a_{0}[a \cdot \cos (\phi)+2 x \cos (t-\phi)-15 a \cdot \cos (2 t-\phi)]}{4\left[r_{0}^{2}-a_{0} \cos (t-\phi)\right]^{5 / 2}}+ \\
& \frac{15 a_{0}^{2}[-2 x+14 a \cdot \cos (t)+14 x \cos (2 t-2 \phi)-a \cdot \cos (t-2 \phi)-25 a \cdot \cos (3 t-2 \phi)]}{16\left[r_{0}^{2}-a_{0} \cos (t-\phi)\right]^{7 / 2}}+ \\
& \frac{105 a_{0}^{3}[a \cdot \cos (\phi)-6 x \cos (t-\phi)+5 a \cdot \cos (2 t-\phi)] \sin (t-\phi)^{2}}{8\left[r_{0}^{2}-a_{0} \cos (t-\phi)\right]^{9 / 2}}+\frac{945 a_{0}^{4}[x-a \cos (t)] \sin (t-\phi)^{4}}{16\left[r_{0}^{2}-a_{0} \cos (t-\phi)\right]^{1 / 2}}
\end{aligned}
$$

The integrand, $f$, varies significantly with the point $(x, y, z)$ as is evident in Figure 16 where, for the case of $a=1$ and $b=0$, the graph of $f$ is shown for the points $(1 / 10,1 / 10,1 / 10),(1,1 / 10,1 / 10),(1,1,1)$ and $(10,1,1)$. The change in the integrand with four sub-divisions of the interval $[0,2 \pi]$ is shown in Figure 17 for the same four points.

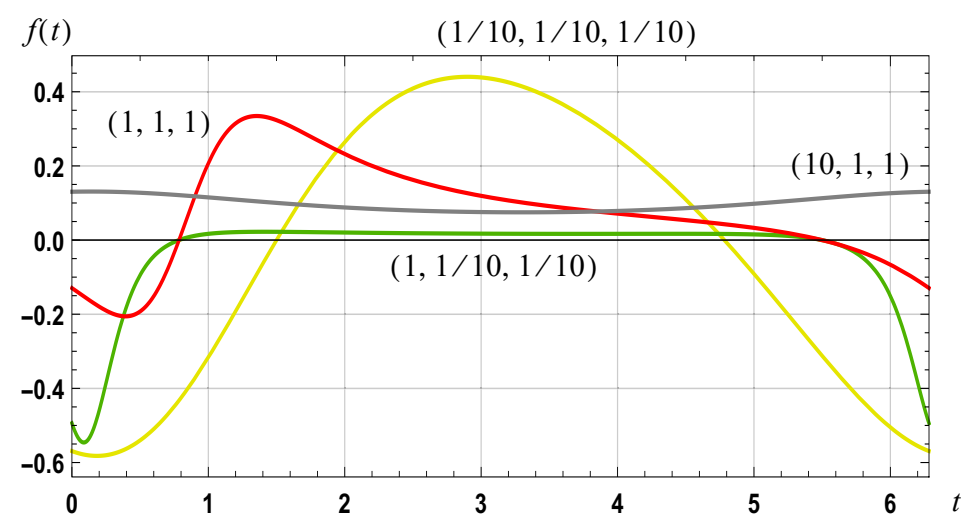

Figure 16. Graph of $f(t)$ for the case of $a=1, b=0$. For the case of $(10,1,1)$ a scaling factor of 10 has been used; for the case of $(1,1 / 10,1 / 10)$ a scaling factor of 0.1 has been used.

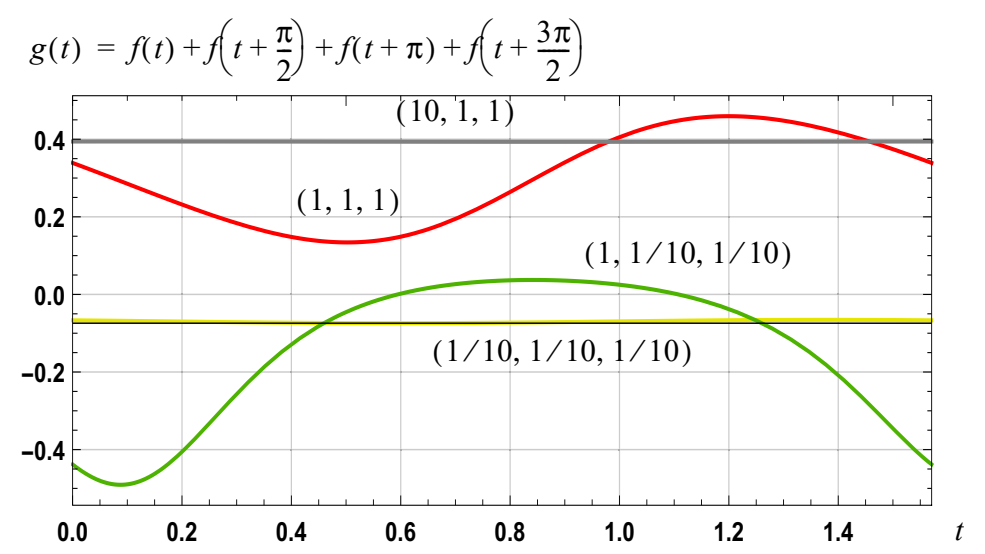

Figure 17. Graph of $g(t)$. For the case of $(10,1,1)$ a scaling factor of 10 has been used; for the case of $(1,1 / 10,1 / 10)$ a scaling factor of 0.1 has been used. 
Indicative results are detailed in Table 9 and the use of four sub-intervals yields, in general, acceptable levels of error apart from the case of points close to the ring of charge where the integrand varies rapidly.

Table 9. Magnitude of relative error in evaluation of $E_{X}(x, y, z)$ using four sub-intervals and eight sub-intervals for the point $(1,0.1,0.1)$.

\begin{tabular}{ccccc}
\hline Order of Approx. $\boldsymbol{n}$ & Point: (0.1, 0.1, 0.1) & $\begin{array}{c}\text { Point: (1, 0.1, 0.1) } \\
\text { (8 Intervals) }\end{array}$ & Point: 90 (1, 1, 1) & Point: (10, 1, 1) \\
\hline 2 & 0.015 & 0.15 & 0.067 & $3.1 \times 10^{-4}$ \\
4 & $2.9 \times 10^{-4}$ & $4.6 \times 10^{-3}$ & $2.4 \times 10^{-2}$ & $5.3 \times 10^{-6}$ \\
8 & $2.6 \times 10^{-8}$ & $2.3 \times 10^{-3}$ & $2.6 \times 10^{-3}$ & $1.1 \times 10^{-9}$ \\
16 & $3.6 \times 10^{-16}$ & $2.9 \times 10^{-5}$ & $2.7 \times 10^{-5}$ & $2.1 \times 10^{-17}$ \\
\hline
\end{tabular}

\section{Conclusions}

This paper has introduced the dual Taylor series which is a natural generalization of the classic Taylor series. Such a series, along with a spline based series, facilitates function and integral approximation with the approximations being summarized in Table 2. In comparison with a antiderivative series, a Taylor series and a dual Taylor series, a spline based series approximation to an integral, in general, yields the highest accuracy for a set order of approximation. A spline based series for an integral has many applications and indicative examples include a series for the exponential function, which coincides with a Padé series, new series for the logarithm function as well as new series for integral defined functions such as the Fresnel Sine integral function. Such series are more accurate, and have larger regions of convergence, than Taylor series based approximations. The spline based series for an integral can be used to define algorithms for highly accurate approximations for the logarithm function, the exponential function, rational numbers to a fractional power and the inverse sine, inverse cosine and inverse tangent functions. Such algorithms can be used, for example, to establish highly accurate approximations for specific irrational numbers such as $\pi$ and Catalan's constant. The use of sub-intervals allows the region of convergence for an integral to be extended. The results presented are not exhaustive and other applications remain to be found.

Acknowledgments: The support of Prof. Zoubir, SPG, Technical University of Darmstadt, Darmstadt, Germany, who hosted a visit where significant work on this paper was completed, is gratefully acknowledged. Prof. Zoubir suggested the use of a set of test functions for a comparison of the integral approximations.

Conflicts of Interest: The author declares no conflict of interest.

\section{Appendix A. Proof of Remainder Expression for Dual Taylor Series}

As $m(t)+q(t)=1$ for $t \in[\alpha, \beta]$, it follows that the remainder function $R_{n}(\alpha, \beta, t)$, as specified by (28), can be written as

$$
\begin{gathered}
R_{n}(\alpha, \beta, t)=f(t)-f_{n}(\alpha, \beta, t)=[m(t)+q(t)] f(t)-\left[m(t) f_{n}(\alpha, t)+q(t) f_{n}(\beta, t)\right] \\
=m(t)\left[f(t)-f_{n}(\alpha, t)\right]+q(t)\left[f(t)-f_{n}(\beta, t)\right]
\end{gathered}
$$

Using the result for the error in a $n$th order Taylor series approximation, as specified in (9), i.e.,

$$
R_{n}(\alpha, t)=\int_{\alpha}^{t} \frac{(t-\lambda)^{n} f^{(n+1)}(\lambda)}{n !} d \lambda, \quad R_{n}(\beta, t)=-\int_{t}^{\beta} \frac{(t-\lambda)^{n} f^{(n+1)}(\lambda)}{n !} d \lambda
$$


the required results follow, namely:

$$
R_{n}(\alpha, \beta, t)=m(t) \int_{\alpha}^{t} \frac{(t-\lambda)^{n} f^{(n+1)}(\lambda)}{n !} d \lambda-q(t) \int_{t}^{\beta} \frac{(t-\lambda)^{n} f^{(n+1)}(\lambda)}{n !} d \lambda, t \in[\alpha, \beta] .
$$

\section{Appendix B. Integral of a Dual Taylor Series with Polynomial Demarcation Functions}

Consider

$$
I_{n}(t)=\int_{\alpha}^{t} f_{n}(\alpha, t, \lambda) d \lambda=\int_{\alpha}^{t}\left[m_{n}(\lambda) f_{n}(\alpha, \lambda)+q_{n}(\lambda) f_{n}(t, \lambda)\right] d \lambda .
$$

Substitution of $f_{n}(\alpha, \lambda)$ and $f_{n}(t, \lambda)$ from (7), along with the definitions $m_{n}(t)=m_{N, n}\left(\frac{t-\alpha}{\beta-\alpha}\right)$ and $q_{n}(t)=m_{N, n}\left(\frac{\beta-t}{\beta-\alpha}\right)$ for the interval $[\alpha, \beta]$, it follows, using the form specified in (21) for $m_{N, n}(t)$, that

$$
\begin{aligned}
I_{n}(t)= & \int_{\alpha}^{t}\left[(n+1) \sum_{u=0}^{n+1} \sum_{v=0}^{n} \frac{(-1)^{u} \cdot(n+v) !}{(n+1-u) ! \cdot u ! \cdot v !} \cdot\left[\frac{\lambda-\alpha}{t-\alpha}\right]^{u+v} \cdot \sum_{k=0}^{n} \frac{(\lambda-\alpha)^{k} f^{(k)}(\alpha)}{k !}\right] d \lambda+ \\
& \int_{\alpha}^{t}\left[(n+1) \sum_{u=0}^{n+1} \sum_{v=0}^{n} \frac{(-1)^{u} \cdot(n+v) !}{(n+1-u) ! \cdot u ! \cdot v !} \cdot\left[\frac{t-\lambda}{t-\alpha}\right]^{u+v} \cdot \sum_{k=0}^{n} \frac{(\lambda-t)^{k} f^{(k)}(t)}{k !}\right] d \lambda
\end{aligned}
$$

Interchanging the order of summations and integration, and evaluation of the integrals, yields the required result:

$$
\begin{aligned}
I_{n}(t) & =(n+1) \sum_{u=0}^{n+1} \sum_{v=0}^{n} \sum_{k=0}^{n} \frac{(-1)^{u} \cdot(n+v) !}{(n+1-u) ! \cdot u ! \cdot v ! \cdot k !} \cdot \frac{(t-\alpha)^{k+1}}{u+v+k+1} \cdot\left[f^{(k)}(\alpha)+(-1)^{k} f^{(k)}(t)\right] \\
& =\sum_{k=0}^{n} c_{n, k} \cdot(t-\alpha)^{k+1} \cdot\left[f^{(k)}(\alpha)+(-1)^{k} f^{(k)}(t)\right]
\end{aligned}
$$

where

$$
c_{n, k}=\frac{n+1}{k !} \cdot \sum_{u=0}^{n+1} \frac{(-1)^{u}}{u ! \cdot(n+1-u) !} \cdot\left[\sum_{v=0}^{n} \frac{(n+v) !}{v !} \cdot \frac{1}{u+v+k+1}\right] .
$$

The remainder is specified by

$$
R_{n}(\alpha, t)=\int_{\alpha}^{t}\left[f(\lambda)-f_{n}(\alpha, t, \lambda)\right] d \lambda=\int_{\alpha}^{t} f(\lambda) d \lambda-I_{n}(t)
$$

which implies

$$
R_{n}^{(1)}(\alpha, t)=f(t)-I_{n}^{(1)}(t)
$$

Differentiation of $I_{n}(t)$, and use of the result $c_{n, 0}=1 / 2$, yields the required result:

$$
\begin{aligned}
R_{n}^{(1)}(\alpha, t)= & c_{n, 0}[f(t)-f(\alpha)]-\sum_{k=1}^{n} c_{n, k}(k+1)(t-\alpha)^{k} f^{(k)}(\alpha)+ \\
& \sum_{k=1}^{n}(-1)^{k+1}(t-\alpha)^{k} f^{(k)}(t)\left[c_{n, k}(k+1)-c_{n, k-1}\right]+c_{n, n}(-1)^{n+1}(t-\alpha)^{n+1} f^{(n+1)}(t)
\end{aligned}
$$




\section{Appendix C. Proof of Spline Based Approximation}

Consider the form specified by (39) for $f_{n}(\alpha, \beta, t)$ and the results:

$$
\begin{aligned}
& h_{n}^{(k)}(t)=\frac{(n+1) !}{(n+1-k) !} \cdot \frac{(t-\beta)^{n+1-k}}{(\beta-\alpha)^{n+1}}, \quad h^{(k)}(\alpha)=\frac{(n+1) !}{(n+1-k) !} \cdot \frac{(-1)^{n+1-k}}{(\beta-\alpha)^{k}}, \\
& g_{n}^{(k)}(t)=\frac{(n+1) !}{(n+1-k) !} \cdot \frac{(t-\alpha)^{n+1-k}}{(\beta-\alpha)^{n+1}}, \quad g^{(k)}(\beta)=\frac{(n+1) !}{(n+1-k) !} \cdot \frac{1}{(\beta-\alpha)^{k}}
\end{aligned}
$$

Using these results, (39) allows the unknown coefficients to be sequentially solved. For example:

$$
\begin{gathered}
f_{n}(\alpha, \beta, \alpha)=f(\alpha) \Rightarrow h_{n}(\alpha) a_{00}=1 \\
f_{n}^{(1)}(\alpha, \beta, \alpha)=f^{(1)}(\alpha) \Rightarrow\left\{\begin{array}{c}
h_{n}^{(1)}(\alpha) a_{00}+h_{n}(\alpha) a_{01}=0 \\
h_{n}(\alpha) a_{10}=1
\end{array}\right. \\
f_{n}^{(2)}(\alpha, \beta, \alpha)=f^{(2)}(\alpha) \Rightarrow\left\{\begin{array}{c}
h_{n}^{(2)}(\alpha) a_{00}+2 h_{n}^{(1)}(\alpha) a_{01}+2 h_{n}(\alpha) a_{02}=0 \\
2 h_{n}^{(1)}(\alpha) a_{10}+2 h_{n}(\alpha) a_{11}=0 \\
2 h_{n}(\alpha) a_{20}=1
\end{array}\right.
\end{gathered}
$$

etc. Sequential solving of the equations yields the result stated in (40).

To rewrite (40) in a form that is similar to the form of a dual Taylor series, both the numerator and denominator expressions can be multiplied by $\sum_{i=0}^{n} \frac{(n+i) !}{i ! \cdot n !} \cdot \frac{(t-\alpha)^{i}}{(\beta-\alpha)^{i}}$ for the first term, and $\sum_{i=0}^{n} \frac{(n+i) !}{i ! \cdot n !} \cdot \frac{(\beta-t)^{i}}{(\beta-\alpha)^{i}}$ for the second, to yield

$$
\begin{aligned}
& f_{n}(\alpha, \beta, t)=\left[\frac{(\beta-t)^{n+1}}{(\beta-\alpha)^{n+1}} \cdot \sum_{i=0}^{n} \frac{(n+i) !}{i ! n !} \cdot \frac{(t-\alpha)^{i}}{(\beta-\alpha)^{i}}\right] \cdot \sum_{k=0}^{n} \frac{(t-\alpha)^{k}}{k !} \cdot f^{(k)}(\alpha) \cdot \frac{\sum_{i=0}^{n-k} \frac{(n+i) !}{i ! \cdot n !} \cdot \frac{(t-\alpha)^{i}}{(\beta-\alpha)^{i}}}{\sum_{i=0}^{n} \frac{(n+i) !}{i ! \cdot n !} \cdot \frac{(t-\alpha)^{i}}{(\beta-\alpha)^{i}}}+ \\
& {\left[\frac{(t-\alpha)^{n+1}}{(\beta-\alpha)^{n+1}} \cdot \sum_{i=0}^{n} \frac{(n+i) !}{i ! \cdot n !} \cdot \frac{(\beta-t)^{i}}{(\beta-\alpha)^{i}}\right] \cdot \sum_{k=0}^{n} \frac{(-1)^{k}(\beta-t)^{k}}{k !} \cdot f^{(k)}(\beta) \cdot \frac{\sum_{i=0}^{n-k} \frac{(n+i) !}{i ! \cdot n !} \cdot \frac{(\beta-t)^{i}}{(\beta-\alpha)^{i}}}{\sum_{i=0}^{n} \frac{(n+i) !}{i ! \cdot n !} \cdot \frac{(\beta-t)^{i}}{(\beta-\alpha)^{i}}}}
\end{aligned}
$$

With the definition of the polynomial demarcation functions, as specified by (23), it follows that

$$
\begin{aligned}
& f_{n}(\alpha, \beta, t)=m_{n}(t) \cdot \sum_{k=0}^{n} \frac{(t-\alpha)^{k}}{k !} \cdot f^{(k)}(\alpha) \cdot\left[\frac{\sum_{i=0}^{n-k} \frac{(n+i) !}{i ! n !} \cdot \frac{(t-\alpha)^{i}}{(\beta-\alpha)^{i}}}{\sum_{i=0}^{n} \frac{(n+i) !}{i ! n !} \cdot \frac{(t-\alpha)^{i}}{(\beta-\alpha)^{i}}}\right]+ \\
& q_{n}(t) \cdot \sum_{k=0}^{n} \frac{(-1)^{k}(\beta-t)^{k}}{k !} \cdot f^{(k)}(\beta) \cdot\left[\frac{\sum_{i=0}^{n-k} \frac{(n+i) !}{i ! n !} \cdot \frac{(\beta-t)^{i}}{(\beta-\alpha)^{i}}}{\sum_{i=0}^{n} \frac{(n+i) !}{i ! n !} \cdot \frac{(\beta-t)^{i}}{(\beta-\alpha)^{i}}}\right]
\end{aligned}
$$

Adding and subtracting one from the bracketed terms, leads to the definitions for $c_{n, k}(t)$ and $d_{n, k}(t)$ as specified by (42) and the required form for $f_{n}(\alpha, \beta, t)$ as stated by (41).

\section{Appendix D. Convergence of Spline Approximation}

Consider the spline approximation defined by (41):

$$
f_{n}(\alpha, \beta, t)=m_{n}(t) \cdot \sum_{k=0}^{n} \frac{(t-\alpha)^{k}}{k !} \cdot f^{(k)}(\alpha) \cdot\left[1+c_{n, k}(t)\right]+q_{n}(t) \cdot \sum_{k=0}^{n} \frac{(t-\beta)^{k}}{k !} \cdot f^{(k)}(\beta) \cdot\left[1+d_{n, k}(t)\right] .
$$


First, assume the Taylor series

$$
\sum_{k=0}^{n} \frac{(t-\alpha)^{k}}{k !} \cdot f^{(k)}(\alpha), \quad \sum_{k=0}^{n} \frac{(t-\beta)^{k}}{k !} \cdot f^{(k)}(\beta)
$$

converge, respectively, over the intervals $\alpha<t<\alpha+\frac{\beta-\alpha}{2}$ and $\alpha+\frac{\beta-\alpha}{2}<t<\beta$. Consistent with (32), a sufficient condition for convergence is for

$$
\lim _{n \rightarrow \infty} \frac{f_{\max }^{(n)}(\beta-\alpha)^{n}}{2^{n} \cdot n !}=0
$$

It then follows that

$$
\sum_{k=0}^{n} \frac{(t-\alpha)^{k}}{k !} \cdot f^{(k)}(\alpha) \cdot\left[1+c_{n, k}(t)\right] \text { and } \sum_{k=0}^{n} \frac{(t-\beta)^{k}}{k !} \cdot f^{(k)}(\beta) \cdot\left[1+d_{n, k}(t)\right]
$$

both converge. To show this consider (42) and $1+c_{n, k}(\delta)$ :

$$
1+c_{n, k}(\delta)=\frac{\sum_{i=0}^{n-k} \frac{(n+i) !}{i ! \cdot n !} \cdot \delta^{i}}{\sum_{i=0}^{n} \frac{(n+i) !}{i ! \cdot n !} \cdot \delta^{i}}, \quad \delta=\frac{(t-\alpha)}{(\beta-\alpha)} .
$$

It is clear that $1+c_{n, k+1}(\delta)$ contains one less term than $1+c_{n, k}(\delta)$ in the numerator and all terms are positive. Thus, $1+c_{n, k+1}(\delta)<1+c_{n, k}(\delta)$ and it is the case that $0<1+c_{n, k}(\delta) \leq 1$ with equality for the case of $k=0$. For $n$ and $\delta$ fixed, $1+c_{n, k}(\delta)$ is a monotonically decreasing series for $k \in\{0, \ldots, n\}$, as is clearly evident in the graphs shown in Figure 5. It then follows, from Abel's test for convergence [22], that the series specified in (161) are convergent.

Second, the issue is the convergence of the spline approximation to the underlying function on the interval $[\alpha, \beta]$. This follows if $\lim _{n \rightarrow \infty} c_{n, k}(\delta)=0$ for $k \in\{0,1, \ldots, n\}$ as the summation is then that of a dual Taylor series. Further, $m_{n}(t)$ and $q_{n}(t)$ approach ideal demarcation functions as $n \rightarrow \infty$. Thus, the requirement is to prove converge of $\left|c_{n, k}(\delta)\right|$ to zero for $0 \leq \delta \leq 1 / 2$. First, using the definition of $c_{n, k}(t)$ specified by $(42)$, it can readily be shown that

$$
\begin{array}{r}
\frac{\partial}{\partial \delta}\left[-c_{n, k}(\delta)\right]=\frac{1}{D} \cdot \sum_{i=n-k+1}^{n} \sum_{j=1}^{n-k} \frac{(n+i) !}{i ! \cdot n !} \cdot \frac{(n+j) !}{j ! \cdot n !} \cdot(i-j) \delta^{i+j-1}+\frac{1}{D} \cdot \sum_{i=n-k+1}^{n} \frac{(n+i) !}{(i-1) ! \cdot n !} \cdot \delta^{i-1}+ \\
\frac{1}{D} \cdot \sum_{i=n-k+1}^{n} \sum_{j=n-k+1}^{n} \frac{(n+i) !}{i ! \cdot n !} \cdot \frac{(n+j) !}{j ! \cdot n !} \cdot(i-j) \delta^{i+j-1}, D=\left[\sum_{i=0}^{n} \frac{(n+i) !}{i ! \cdot n !} \cdot \delta^{i}\right]^{2}
\end{array}
$$

and it then follows that $\frac{\partial}{\partial \delta}\left[-c_{n, k}(\delta)\right]>0$ as the first two summations comprise only of positive terms and the last double summation equals zero as the off-diagonal terms can be paired with one term being the negative of the another. Thus, for $n$ and $k$ fixed

$$
\left|c_{n, k}\left(\delta_{1}\right)\right|<\left|c_{n, k}\left(\delta_{2}\right)\right|
$$

when $\delta_{1}<\delta_{2}$. Hence, if $\lim _{n \rightarrow \infty} c_{n, k}(\delta)=0$ for $\delta=1 / 2$, then the proof is complete. For $\delta=1 / 2$, it is the case that the denominator term for $c_{n, k}(1 / 2)$ simplifies according to

$$
\sum_{i=0}^{n} \frac{(n+i) !}{i ! \cdot n !} \cdot \frac{1}{2^{i}}=2^{n}
$$


It then follows that

$$
\begin{aligned}
\left|c_{n, k}(1 / 2)\right| & =\frac{1}{2^{n}} \cdot\left[\frac{(2 n-k+1) !}{(n-k+1) ! \cdot n !} \cdot \delta^{n-k+1}+\frac{(2 n-k+2) !}{(n-k+2) ! n !} \cdot \delta^{n-k+2}+\ldots+\frac{(2 n) !}{n ! \cdot n !} \cdot \delta^{n}\right] \mid \delta=1 / 2 \\
& \left.\approx \frac{(2 n-k+1) !}{(n-k+1) ! n !} \cdot \frac{\delta^{n-k+1}}{2^{n}}\left[1+2 \delta+2^{2} \delta^{2}+\ldots+2^{k-1} \delta^{k-1}\right]\right|_{\delta=1 / 2}
\end{aligned}
$$

assuming $n \gg k$. Hence:

$$
\left|c_{n, k}(1 / 2)\right| \approx \frac{(2 n-k+1) !}{(n-k+1) \cdot n !} \cdot \frac{k \cdot 2^{k-1}}{2^{2 n}}, \quad k \ll n
$$

Using Stirling's formula, $n ! \approx \sqrt{2 \pi} \cdot n^{n+1 / 2} \cdot e^{-n}$, yields

$$
\left|c_{n, k}(1 / 2)\right| \approx \frac{k}{\sqrt{\pi}} \cdot \frac{1}{\sqrt{n}}
$$

which clearly converges to zero as $n$ increases for all fixed values of $k$. This completes the proof.

\section{Appendix E. Proof: Integral Approximation Based on Spline Approximation}

The spline based integral approximation, as specified by (49) and (50), arises from rewriting (40) in the form:

$$
\begin{aligned}
f_{n}(\alpha, \beta, t)= & \frac{1}{(\beta-\alpha)^{n+1}} \cdot \sum_{k=0}^{n} \frac{f^{(k)}(\alpha)}{k !} \cdot \sum_{i=0}^{n-k} \frac{(n+i) !}{i ! \cdot n !} \cdot \frac{(\beta-t)^{n+1}(t-\alpha)^{i+k}}{(\beta-\alpha)^{i}}+ \\
& \frac{1}{(\beta-\alpha)^{n+1}} \cdot \sum_{k=0}^{n} \frac{(-1)^{k}}{k !} \cdot f^{(k)}(\beta) \cdot \sum_{i=0}^{n-k} \frac{(n+i) !}{i ! \cdot n !} \cdot \frac{(t-\alpha)^{n+1}(\beta-t)^{i+k}}{(\beta-\alpha)^{i}}
\end{aligned}
$$

Integration of this expression over the interval $[\alpha, \beta]$ then yields:

$$
\begin{aligned}
\int_{\alpha}^{\beta} f_{n}(\alpha, \beta, \lambda) d \lambda= & \frac{1}{(\beta-\alpha)^{n+1}} \cdot \sum_{k=0}^{n} \frac{f^{(k)}(\alpha)}{k !} \cdot \sum_{i=0}^{n-k} \frac{(n+i) !}{i ! \cdot n !} \cdot \frac{I_{1}}{(\beta-\alpha)^{i}}+ \\
& \frac{1}{(\beta-\alpha)^{n+1}} \cdot \sum_{k=0}^{n} \frac{(-1)^{k}}{k !} \cdot f^{(k)}(\beta) \cdot \sum_{i=0}^{n-k} \frac{(n+i) !}{i ! \cdot n !} \cdot \frac{I_{2}}{(\beta-\alpha)^{i}}
\end{aligned}
$$

where

$$
I_{1}=\int_{\alpha}^{\beta}(\beta-\alpha)^{n+1} \cdot(\lambda-\alpha)^{i+k} d \lambda, \quad I_{2}=\int_{\alpha}^{\beta}(\lambda-\alpha)^{n+1} \cdot(\beta-\lambda)^{i+k} d \lambda .
$$

A change of variable $r=\frac{\lambda-\alpha}{\beta-\alpha}$ yields

$$
\begin{aligned}
& I_{1}=(\beta-\alpha)^{n+2+i+k} \int_{0}^{1}(1-r)^{n+1} \cdot r^{i+k} d r=(\beta-\alpha)^{n+2+i+k} \cdot B(i+k+1, n+2), \\
& I_{2}=(\beta-\alpha)^{n+2+i+k} \int_{0}^{1} r^{n+1} \cdot(1-r)^{i+k} d r=(\beta-\alpha)^{n+2+i+k} \cdot B(n+2, i+k+1),
\end{aligned}
$$

where $B$ is the Beta function

$$
B(a, b)=\int_{0}^{1} x^{a-1}(1-x)^{b-1} d x
$$


As

$$
B(m, n)=B(n, m)=\frac{\Gamma(m) \Gamma(n)}{\Gamma(m+n)}=\frac{(m-1) ! \cdot(n-1) !}{(m+n-1) !}, m, n \in\{1,2, \ldots\},
$$

it follows that

$$
I_{1}=I_{2}=(\beta-\alpha)^{n+2+i+k} \cdot \frac{(n+1) ! \cdot(i+k) !}{(n+i+k+2) !} .
$$

Substitution yields the required result:

$$
\int_{\alpha}^{\beta} f_{n}(\alpha, \beta, \lambda) d \lambda=(n+1) \cdot \sum_{k=0}^{n} \frac{(\beta-\alpha)^{k+1}}{k !} \cdot\left[f^{(k)}(\alpha)+(-1)^{k} \cdot f^{(k)}(\beta)\right] \cdot \sum_{i=0}^{n-k} \frac{(n+i) !}{i !} \cdot \frac{(i+k) !}{(n+i+k+2) !} .
$$

Appendix E.1. Second Form

By directly solving for the coefficients associated with a $n$th order spline approximation to a function over the interval $[\alpha, \beta]$, i.e., solving for $d_{0}, \ldots, d_{2 n+1}$ in the following approximation function

$$
f_{n}(\alpha, \beta, t)=\sum_{i=0}^{2 n+1} d_{i}(t-\alpha)^{i}, \quad f_{n}^{(k)}(\alpha, \beta, \alpha)=f^{(k)}(\alpha), \quad f_{n}^{(k)}(\alpha, \beta, \beta)=f^{(k)}(\beta), \quad k \in\{0,1, \ldots, n\}
$$

and then integrating the approximation over $[\alpha, t]$, yields

$$
\int_{\alpha}^{t} f_{n}(\alpha, t, \lambda) d \lambda=\sum_{k=0}^{n} c_{n, k} \cdot(t-\alpha)^{k+1} \cdot\left[f^{(k)}(\alpha)+(-1)^{k} f^{(k)}(t)\right],
$$

where the coefficients form the array:

$$
\begin{array}{lcclllllll}
n=0 & \frac{1}{2} & & & & & & \\
n=1 & \frac{1}{2} & \frac{1}{12} & & & & & \\
n=2 & \frac{1}{2} & \frac{1}{10} & \frac{1}{120} & & & & \\
n=3 & \frac{1}{2} & \frac{3}{28} & \frac{1}{84} & \frac{1}{1680} & & & \\
n=4 & \frac{1}{2} & \frac{1}{9} & \frac{1}{72} & \frac{1}{1008} & \frac{1}{30240} & & \\
n=5 & \frac{1}{2} & \frac{5}{44} & \frac{1}{66} & \frac{1}{792} & \frac{1}{15840} & \frac{1}{665280} & \\
n=6 & \frac{1}{2} & \frac{3}{26} & \frac{5}{312} & \frac{5}{3432} & \frac{1}{11440} & \frac{1}{308880} & \frac{1}{17,297,280} \\
& k=0 & k=1 & & & & &
\end{array}
$$

The explicit expression $c_{n, k}=\frac{n !}{(n-k) !(k+1) !} \cdot \frac{(2 n+1-k) !}{2 \cdot(2 n+1) !}$ can then be inferred. A direct proof of the equality between this coefficient expression and the expression implicit in (177), i.e., a direct proof of

$$
\frac{n+1}{k !} \sum_{i=0}^{n-k} \frac{(n+i) !}{i !} \cdot \frac{(k+i) !}{(n+k+i+2) !}=\frac{n !}{(n-k) !(k+1) !} \cdot \frac{(2 n+1-k) !}{2 \cdot(2 n+1) !}
$$

remains elusive.

Appendix E.2. Remainder

The remainder term, specified by (53), arises from differentiation of (48) which yields

$$
\begin{aligned}
f(t)= & \frac{f(t)}{2}+\frac{f(\alpha)}{2}+\sum_{k=1}^{n} c_{n, k}(k+1)(t-\alpha)^{k} \cdot\left[f^{(k)}(\alpha)+(-1)^{k} f^{(k)}(t)\right]+ \\
& \sum_{k=0}^{n} c_{n, k}(t-\alpha)^{k+1}(-1)^{k} f^{(k+1)}(t)+R_{n}^{(1)}(\alpha, t)
\end{aligned}
$$


as $c_{n, 0}=1 / 2$. A change of index $u=k+1$ in the second summation yields

$$
\begin{aligned}
-R_{n}^{(1)}(\alpha, t)= & \frac{-f(t)}{2}+\frac{f(\alpha)}{2}+\sum_{k=1}^{n} c_{n, k}(k+1)(t-\alpha)^{k} f^{(k)}(\alpha)+ \\
& \sum_{k=1}^{n}(t-\alpha)^{k}(-1)^{k} f^{(k)}(t)\left[(k+1) c_{n, k}-c_{n, k-1}\right]+c_{n, n}(-1)^{n}(t-\alpha)^{n+1} f^{(n+1)}(t)
\end{aligned}
$$

Using the definition of $c_{n, k}$, as specified by (51), it follows that

$$
(k+1) c_{n, k}-c_{n, k-1}=-(k+1) c_{n, k} \cdot \frac{n+1}{n-k+1}
$$

and the required result follows, i.e.,

$$
\begin{aligned}
R_{n}^{(1)}(\alpha, t)= & -\sum_{k=0}^{n} c_{n, k}(k+1)(t-\alpha)^{k} \cdot\left[f^{(k)}(\alpha)+(-1)^{k+1} \cdot \frac{n+1}{n-k+1} \cdot f^{(k)}(t)\right]+ \\
& c_{n, n}(-1)^{n+1}(t-\alpha)^{n+1} f^{(n+1)}(t)
\end{aligned}
$$

\section{Appendix F. Proof of Series Approximation for the Exponential Function}

First, the integral of the exponential function is well known, i.e.,

$$
\int_{0}^{t} e^{\lambda} d \lambda=e^{t}-1
$$

Second, a spline based integral approximation, as specified by (49) and (51), for the integral of the exponential function yields

$$
\begin{aligned}
\int_{0}^{t} e^{\lambda} d \lambda & \approx \sum_{k=0}^{n} c_{n, k} t^{k+1}\left[1+(-1)^{k} e^{t}\right] \\
& =\left[c_{n, 0} t+c_{n, 1} t^{2}+\ldots+c_{n, n} t^{n+1}\right]+e^{t}\left[c_{n, 0} t-c_{n, 1} t^{2}+\ldots+(-1)^{n} c_{n, n} t^{n+1}\right]
\end{aligned}
$$

Equating the right hand side of these two equations results in the required approximation

$$
e^{t} \approx \frac{1+c_{n, 0} t+c_{n, 1} t^{2}+\ldots+c_{n, n} t^{n+1}}{1-c_{n, 0} t+c_{n, 1} t^{2}+\ldots+(-1)^{n+1} c_{n, n} t^{n+1}} .
$$

The result stated in (75), for the case of $n \rightarrow \infty$, arises from the result (54): $c_{\infty, k}=\frac{1}{2^{k+1}} \cdot \frac{1}{(k+1) !}$.

The series convergence is based on the convergence of (49) for the case of an integrand of $f(t)=\exp (t)$. A sufficient condition for this is convergence of a spline based approximation for $\exp (t)$ over the interval $[0, t]$. Consistent with (47), a sufficient condition for this is for $\lim _{n \rightarrow \infty} \frac{t^{n}}{2^{n} \cdot n !} \cdot f_{\max }^{(n)}=0$. On the interval $[0, t], f_{\max }^{(n)}=e^{t}$ and, for $t$ fixed:

$$
\lim _{n \rightarrow \infty} \frac{t^{n}}{2^{n} \cdot n !} \cdot f_{\max }^{(n)}=\lim _{n \rightarrow \infty} \frac{t^{n} e^{t}}{2^{n} \cdot n !}=0
$$

as required. 


\section{Appendix G. Proof of Natural Logarithm Approximation}

Consider the integral of $\ln (t)$ :

$$
\int_{1}^{t} \ln (\lambda) d \lambda=t \ln (t)-t+1
$$

The $n$th order based spline series approximation to the integral of $\ln (t)$, as defined by (49), is

$$
\int_{1}^{t} \ln (\lambda) d \lambda \approx \sum_{k=0}^{n} c_{n, k}(t-1)^{k+1}\left[f^{(k)}(1)+(-1)^{k} f^{(k)}(t)\right]
$$

where $f(t)=\ln (t)$ and $f^{(k)}(t)=\frac{(-1)^{k+1}(k-1) !}{t^{k}}, k \in\{1,2, \ldots\}$. Thus:

$$
\int_{1}^{t} \ln (\lambda) d \lambda \approx c_{n, 0}(t-1) \ln (t)+(t-1) \sum_{k=1}^{n} c_{n, k}(-1)^{k+1}(k-1) !(t-1)^{k}\left[1+\frac{(-1)^{k}}{t^{k}}\right] .
$$

As $c_{n, 0}=1 / 2$, equating the right hand sides of this equation and (190) yields the approximation

$$
t \ln (t)-t+1 \approx \frac{(t-1)}{2} \cdot \ln (t)+(t-1) \sum_{k=1}^{n} c_{n, k}(-1)^{k+1}(k-1) !(t-1)^{k}\left[1+\frac{(-1)^{k}}{t^{k}}\right] .
$$

Simplification yields the required result:

$$
\ln (t) \approx \frac{2(t-1)}{t+1}\left[1+\sum_{k=1}^{n} c_{n, k}(-1)^{k+1}(k-1) !(t-1)^{k}\left[1+\frac{(-1)^{k}}{t^{k}}\right]\right] .
$$

\section{Appendix H. Iterative Algorithm for Number to a Fractional Power}

Consider the integral result

$$
\int_{z_{0}^{h}}^{x_{o}} \frac{1}{h t^{1-1 / h}} d t=\left.t^{1 / h}\right|_{z_{0}^{h}} ^{x_{o}}=x_{o}^{1 / h}-z_{0}
$$

With

$$
f(t)=\frac{1}{h t^{1-1 / h}}, \quad f^{(k)}(t)=\frac{(-1)^{k}}{h t^{k+1-1 / h}} \cdot \prod_{j=1}^{k}\left(j-\frac{1}{h}\right)=\frac{(-1)^{k+1}}{t^{k+1-1 / h}} \cdot \prod_{j=0}^{k}\left(j-\frac{1}{h}\right),
$$

where the last expression for $f^{(k)}(t)$ is valid for $k \in\{1,2, \ldots\}$, it follows, based on a $n$th order spline approximation as defined by (49) and (51), that

$$
\begin{aligned}
\int_{z_{i}^{h}}^{x_{o}} \frac{1}{h t^{1-1 / h}} d t & \approx \sum_{k=0}^{n} c_{n, k}(-1)^{k+1}\left(x_{o}-z_{i}^{h}\right)^{k+1} \prod_{j=0}^{k}\left(j-\frac{1}{h}\right) \cdot\left[\frac{1}{z_{i}^{k h+h-1}}+(-1)^{k} \cdot \frac{x_{o}^{1 / h}}{x_{o}^{k+1}}\right] \\
& =u_{i+1}+v_{i+1} x_{o}^{1 / h}
\end{aligned}
$$


Here

$$
\begin{aligned}
& u_{i+1}=\sum_{k=0}^{n} c_{n, k}(-1)^{k+1}\left(x_{o}-z_{i}^{h}\right)^{k+1}\left[\prod_{j=0}^{k}\left(j-\frac{1}{h}\right)\right] \cdot \frac{1}{z_{i}^{k+h+1}}, \\
& v_{i+1}=-\sum_{k=0}^{n} c_{n, k}\left(x_{o}-z_{i}^{h}\right)^{k+1}\left[\prod_{j=0}^{k}\left(j-\frac{1}{h}\right)\right] \cdot \frac{1}{x_{o}^{k+1}}
\end{aligned}
$$

As

$$
\int_{z_{i}^{h}}^{x_{o}} \frac{1}{h t^{1-1 / h}} d t=x_{o}^{1 / h}-z_{i} \approx u_{i+1}+v_{i+1} x_{o}^{1 / h}
$$

it then follows that an updated approximation for $x_{0}^{1 / h}$ is

$$
x_{o}^{1 / h} \approx \frac{z_{i}+u_{i+1}}{1-v_{i+1}} .
$$

This approximation is $z_{i+1}$ and is the basis for the next lower integral limit of $z_{i+1}^{h}$. Thus:

$$
z_{i+1}=\frac{z_{i}+u_{i+1}}{1-v_{i+1}}
$$

\section{Appendix I. Proof: ArcCos, ArcSin and ArcTangent Approximation}

Consider a point $(x, y)$ on the first quadrant of a unit circle, as illustrated in Figure A1, which defines an angle $\theta$. The relationships $\theta(y)=\operatorname{asin}(y)$, and $\frac{d}{d y} \theta(y)=\frac{1}{\sqrt{1-y^{2}}}$, imply that the path length around the unit circle defined by the angle of $\theta / 2^{i}$, to the point $\left(x_{0}, y_{0}\right)$, is

$$
\frac{\theta}{2^{i}}=\int_{0}^{y_{0}} \frac{1}{\sqrt{1-\lambda^{2}}} d \lambda
$$

where

$$
y_{o}=\sin \left[\frac{\theta}{2^{i}}\right]=\cos \left[\frac{\pi}{2}-\frac{\theta}{2^{i}}\right], x_{o}=\cos \left[\frac{\theta}{2^{i}}\right]=\sin \left[\frac{\pi}{2}-\frac{\theta}{2^{i}}\right]
$$

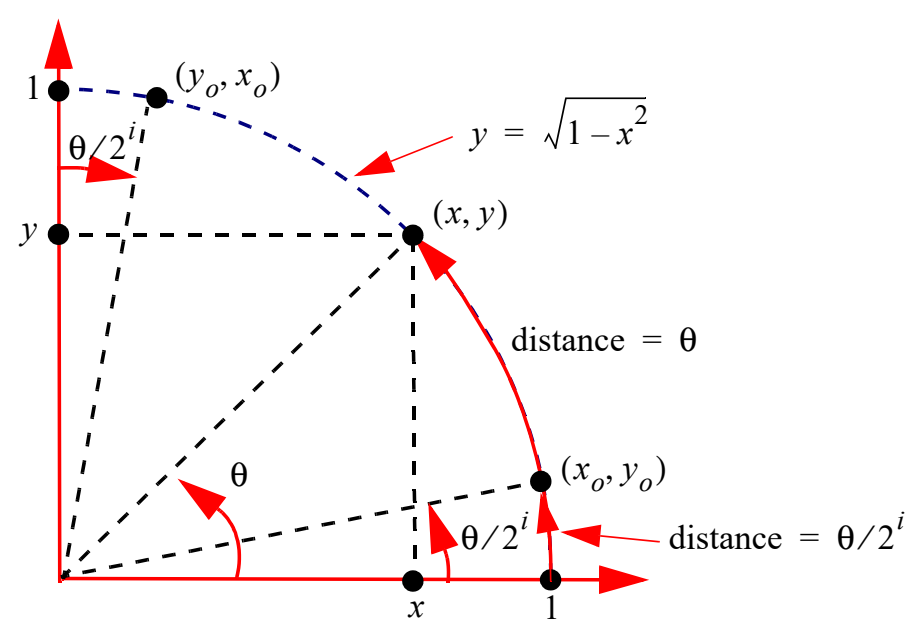

Figure A1. Geometry, based on the unit circle, for defining $\left(x_{0}, y_{0}\right)$ based on the angle $\theta / 2^{i}$. 
To relate $y_{0}$ to $x$ and $y$, consider the well known half angle results for the first quadrant:

$$
\begin{aligned}
& \tan (\theta / 2)=\sqrt{\frac{1-\cos (\theta)}{1+\cos (\theta)}}=\frac{\sin (\theta)}{1+\cos (\theta)}, \\
& \sin (\theta / 2)=\frac{1}{\sqrt{2}} \cdot \sqrt{1-\cos (\theta)}, \quad \cos (\theta / 2)=\frac{1}{\sqrt{2}} \cdot \sqrt{1+\cos (\theta)}
\end{aligned}
$$

It then follows that

$$
\tan (\theta / 4)=\frac{\sin (\theta / 2)}{1+\cos (\theta / 2)}
$$

and iteration leads to the algorithm:

$$
t_{i}=\frac{s_{i-1}}{1+c_{i-1}}, \quad s_{i}=\frac{1}{\sqrt{2}} \cdot \sqrt{1-c_{i-1}}, \quad c_{i}=\frac{1}{\sqrt{2}} \cdot \sqrt{1+c_{i-1}},
$$

where $t_{i}=\tan \left(\theta / 2^{i}\right), s_{i}=\sin \left(\theta / 2^{i}\right), c_{i}=\cos \left(\theta / 2^{i}\right), s_{0}=\sin (\theta)=y$ and $c_{0}=\cos (\theta)=x$. It then follows that

$$
t_{i}=\tan \left(\theta / 2^{i}\right)=\frac{y_{o}}{x_{o}}
$$

Using the notation $\mathrm{g}_{i}(x)=t_{i}$, it follows that

$$
y_{0}=\sqrt{1-y_{0}^{2}} \cdot g_{i}(x) \Rightarrow y_{0}=\sqrt{\frac{g_{i}^{2}(x)}{1+g_{i}^{2}(x)}}
$$

and

$$
\theta=2^{i} \cdot \int_{0}^{\frac{g_{i}(x)}{\sqrt{1+g_{i}^{2}(x)}}} \frac{1}{\sqrt{1-\lambda^{2}}} d \lambda
$$

as required. The alternative definition for $g_{i}(x)$, as defined by (100), arises from a consideration of the results for $t_{i}, i \in\{0,1, \ldots\}$ :

$$
\begin{aligned}
& g_{0}(x)=\frac{y}{x}=\frac{4 x \sqrt{1-x^{2}}}{4 x^{2}}=\frac{n_{0}}{d_{0}} \\
& g_{1}(x)=\frac{y}{1+x}=\frac{2 \sqrt{1-x^{2}}}{2(1+x)}=\frac{n_{1}}{d_{1}} \\
& g_{2}(x)=\frac{\sqrt{2-2 x}}{2+\sqrt{2} \sqrt{1+x}}=\frac{\sqrt{2-\sqrt{d_{0}}}}{2+\sqrt{d_{1}}}=\frac{n_{2}}{d_{2}} \\
& g_{3}(x)=\frac{\sqrt{2-\sqrt{2} \sqrt{1+x}}}{2+\sqrt{2+\sqrt{2} \sqrt{1+x}}}=\frac{\sqrt{2-\sqrt{d_{1}}}}{2+\sqrt{d_{2}}}=\frac{n_{3}}{d_{3}} \\
& g_{4}(x)=\frac{\sqrt{2-\sqrt{2+\sqrt{2} \sqrt{1+x}}}}{2+\sqrt{2+\sqrt{2+\sqrt{2} \sqrt{1+x}}}}=\frac{\sqrt{2-\sqrt{d_{2}}}}{2+\sqrt{d_{3}}}=\frac{n_{4}}{d_{4}}
\end{aligned}
$$

The iterative formula defined by (100) and (101) is clearly evident.

\section{References}

1. Burton, D.M. The History of Mathematics: An Introduction; McGraw-Hill: New York, NY, USA, 2005.

2. Katz, V.J. A History of Mathematics: An Introduction; Addison-Wesley: Boston, MA, USA, 2009.

3. Thomson, B.S. The natural integral on the real line. Scientiae Mathematicae Japonicae 2007, 67, 717-729.

4. Thomson, B.S. Theory of the Integral. Available online: http://classicalrealanalysis.info/com/documents/totiscreen-Feb9-2013.pdf (accessed on 17 December 2018).

5. Botsko, M.W. A fundamental theorem of calculus that applies to all Riemann integrable functions. Math. Mag. 1991, 64, 347-348. [CrossRef]

6. Spivak, M. Calculus; Publish or Perish: Houston, TX, USA, 1994. 
7. Gradsteyn, I.S.; Ryzhik, I.M. Tables of Integrals, Series and Products; Academic Press: Cambridge, MA, USA, 1980.

8. Horowitz, D. Tabular integration by parts. Coll. Math. J. 1990, 21, 307-311. [CrossRef]

9. Olver, F.W.J. Asymptotics and Special Functions; Academic Press: Cambridge, MA, USA, 1974; Chapter 1.

10. Stenger, F. The asymptotic approximation of certain integrals. SIAM J. Math. Anal. 1970, 1, 392-404. [CrossRef]

11. Champeney, D.C. A Handbook of Fourier Theorems; Cambridge University Press: Cambridge, UK, 1987.

12. Pinkus, A. Weierstrass and Approximation Theory. J. Approx. Theory 2000, 107, 1-66. [CrossRef]

13. Davis, P.J.; Rabinowitz, P. Methods of Numerical Integration; Academic Press: Cambridge, MA, USA, 1984.

14. Howard, R.M. Antiderivative series for differentiable functions. Int. J. Math. Educ. Sci. Technol. 2004, 35, 725-731. [CrossRef]

15. Struik, D.J. A Source Book in Mathematics 1200-1800; Princeton University Press: Princeton, NJ, USA, 1986; pp. 328-332.

16. Kountourogiannis, D.; Loya, P. A derivation of Taylor's formula with integral remainder. Math. Mag. 2003, 76, 217-219. [CrossRef]

17. Baker, G.A. Essentials of Pade Approximants; Academic Press: Cambridge, MA, USA, 1975.

18. Finch, S.R. Mathematical Constants, Encyclopedia of Mathematics and its Applications; Cambridge University Press: Cambridge, UK, 2003; Volume 94, pp. 56-57.

19. Weisstein, E.W. Pi Formulas, MathWorld-A Wolfram Web Resource. Available online: http://mathworld. wolfram.com/PiFormulas.html (accessed on 17 December 2018).

20. Abramowitz, M.; Stegun, I.A. Handbook of Mathematical Functions with Formulas, Graphs and Mathematical Tables; Dover: Mineola, NY, USA, 1965.

21. Spiegel, M.R.; Lipshutz, S.; Liu, J. Mathematical Handbook of Formulas and Tables; McGraw Hill: New York, NY, USA, 2009.

22. Whittaker, E.T.; Watson, G.N. A Course of Modern Analysis; Cambridge University Press: Cambridge, UK, 1920.

(C) 2019 by the author. Licensee MDPI, Basel, Switzerland. This article is an open access article distributed under the terms and conditions of the Creative Commons Attribution (CC BY) license (http://creativecommons.org/licenses/by/4.0/). 